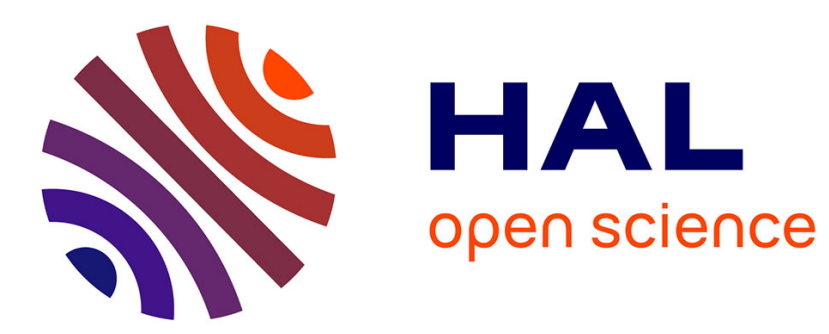

\title{
Galerkin-free model reduction for fluid-structure interaction using proper orthogonal decomposition
}

Vilas Shinde, Elisabeth Longatte, Franck Baj, Yannick Hoarau, Marianna

Braza

\section{- To cite this version:}

Vilas Shinde, Elisabeth Longatte, Franck Baj, Yannick Hoarau, Marianna Braza. Galerkin-free model reduction for fluid-structure interaction using proper orthogonal decomposition. Journal of Computational Physics, 2019, 396, pp.579-595. 10.1016/j.jcp.2019.06.073 . hal-02271516

\section{HAL Id: hal-02271516 https://hal.science/hal-02271516}

Submitted on 26 Aug 2019

HAL is a multi-disciplinary open access archive for the deposit and dissemination of scientific research documents, whether they are published or not. The documents may come from teaching and research institutions in France or abroad, or from public or private research centers.
L'archive ouverte pluridisciplinaire HAL, est destinée au dépôt et à la diffusion de documents scientifiques de niveau recherche, publiés ou non, émanant des établissements d'enseignement et de recherche français ou étrangers, des laboratoires publics ou privés. 


\title{
Galerkin-free model reduction for fluid-structure interaction using proper orthogonal decomposition
}

\author{
Vilas Shinde $^{\mathrm{a}, *}$, Elisabeth Longatte ${ }^{\mathrm{a}}$, Franck Baj ${ }^{\mathrm{a}}$, Yannick Hoarau ${ }^{\mathrm{c}}$, Marianna Braza ${ }^{\mathrm{b}}$ \\ ${ }^{a} I M S I A$, UMR 9219 EDF-CNRS-CEA-ENSTA ParisTech, France \\ ${ }^{b} I M F T, U M R$ CNRS/INPT N 5502, France \\ ${ }^{c}$ University of Strasbourg, ICube - CNRS, Strasbourg, France
}

\begin{abstract}
A Galerkin-free model reduction approach for fluid-structure interaction (FSI) is presented in this article. The reduced order model (ROM) is based on proper orthogonal decomposition (POD), where a reduced basis is formed using energy dominant POD modes. The reduced basis also consists of characteristics POD time modes that are derived from the POD time modes (coefficients) by using their periodicity. In addition to flow variables, the solution state vector comprises the mesh deformation, taking into account the structural deformation in FSI. A ROM solution is obtained by performing a linear interpolation of the reduced basis for changing operating/control parameters. The proposed Galerkin-free POD-ROM approach is demonstrated in terms of two test cases: a canonical case study of vortex-induced vibration (VIV) of a cylinder at Reynolds number $R e=100$, where simulations are performed for various structural-to-fluid mass ratios; and a shock wave boundary layer induced panel flutter. For the second case, we use previously computed high-fidelity simulations, considering only the effect of panel thickness on the aeroelastic coupling between the flexible panel and shock wave boundary layer interaction (SWBLI); the inflow is at Mach 2 and Reynolds number based on panel length $R e_{a}=50000$. The presented Galerking-free ROM procedure is clean and robust for large mesh deformations, in addition to a significantly lower cost of computation compared to the FSI high-fidelity simulations.
\end{abstract}

Keywords: Reduced-order modeling, fluid-structure interaction, proper orthogonal decomposition, Navier-Stokes equations, panel flutter

\section{Introduction}

Computational fluid dynamics (CFD) can be a costly endeavor, particularly for the simulations involving large scale real world problems [1]. Reduced-order models (ROM) can provide computationally inexpensive possibility to perform same computations with a minimum complexity, keeping the essential features of

\footnotetext{
* Corresponding author

Email address: vilas.shinde@polytechnique.edu (Vilas Shinde)
} 
the system intact. Although ROM were initially developed in the context of dynamic systems and control theory, now they are essential in many disciplines. In the context of systems and control theory, the method of balanced truncation [2, 3] is based on the controllability and observability of a control system, while the Hankel-norm reduction [4] method is applied for the approximation of transfer functions. The proper orthogonal decomposition (POD) or Karhunen-Loève expansion is commonly used in fluid dynamics [5, 6] in order to derive ROM as well as to investigate the flow structures and stability. The POD basis provides as an optimal choice, in terms of minimization of the quadratic errors, in order to form a reduced basis [7, which is used for the dimensionality reduction. A traditional procedure to derive a ROM is the Galerkin projection of Navier-Stokes equations (NSE) on the POD time-invariant reduced basis, leading to a reduced system of ordinary differential equations (ODE). The reduced set of ODE is then time integrated to obtain the space-invariant POD modes (also called POD time coefficients). An approximate solution is readily obtained using the POD reduced basis functions.

In addition to the numerical issues associated with the Galerkin ROM, the dynamically moving mesh is a major difficulty in constructing a ROM for fluid-structure interaction (FSI) problems In general for FSI, two separate ROM are derived, one for the fluid domain and other for the structural domain. The two ROM are then coupled to form an FSI-ROM [8, 9, 10, 11. This procedure has been beneficial in terms of reducing the number of parameters and also keeping the two ROM separate. In several other cases, the interface between the fluid and solid domains is also modeled [10].

The FSI-ROM are prominently developed for the aero-elastic flutter [12] and haemodynamics applications [10]. The aeroelastic flutter is also considered as a weakly coupled FSI problem, and it has been studied using FSI-ROM in Silva and Bartels [13, Beran et al. 9] and Raveh [14, to name a few. The recent advancements of ROM for aerodynamic applications are discussed in Lucia et al. [1] and Raveh [14, they include mainly the ROM based on POD, Volterra series and method of Harmonic balance. Contrary to the weekly coupled FSI, the blood flow through arteries and heart represents a strongly coupled FSI problem. where the ROM are expected to provide simplistic models [15, 16, 10].

The influence of mesh deformation on the POD modes has been studied in Anttonen et al. [17, where a multi-POD technique has been adopted in order to select the POD reduced basis depending on the grid displacement; furthermore, the multi-POD was applied to a flow around a pitching and plunging airfoil [18]. The blended POD/ROM based on a forced deformation analysis resulted in an accurate and effective FSIROM. A Galerkin POD-ROM for an FSI is developed in Bourguet et al. [19] for small imposed domain deformations in order to capture the transitional features of a compressible transonic flow. Iemma and Gennaretti 20] developed a ROM using a transonic full-potential aerodynamic model along with its transfer functions and a structural dynamic operator for the fluid-structure coupling. A general framework for constructing an optimization oriented ROM is presented in Bui-Thanh et al. 21, with an example of a subsonic blade row. An FSI-ROM for a steady FSI problem has been presented in Lassila and Rozza 22], 
where a free-boundary problem has been reduced to a low-dimensional parameter space.

The present work builds on Shinde et al. 23, where a Galerkin-free model reduction approach was proposed for Navier-Stokes equations. The Galerkin-free ROM approach treats the solution state variables separately, thus it can be conveniently used for multi-physics problems. In this effort, we extend the Galerkin-free ROM approach for FSI problems, treating large mesh deformations. The presented model reduction technique is demonstrated using two case studies: a two-dimensional vortex induced vibration in a cylinder and a three-dimensional shock wave boundary layer induced panel flutter. Section 2 describes the mathematical formulation of the Galerkin-free ROM procedure including a posteriori error estimate. The results and discussion of the test cases are presented in Sec. 3. while some concluding remarks are provided in Sec. 4 .

\section{Mathematical formulation}

The computational fluid dynamics (CFD) simulations are considered as the high-fidelity model (HFM), where a solution state vector comprises the mesh deformation in addition to the solution of Navier-Stokes equations. Let the solution state vector $s=s(\chi, t ; \eta)$ be spanned on the a time dependent space $\chi \in \Omega$, which is mapped by using a mapping function $\boldsymbol{\xi}$ on a time independent space $\boldsymbol{x} \in \Omega$, where $\Omega \subset \mathbb{R}^{3}$. The time is denoted by $t \in T$, where $T \subset \mathbb{R}$. The variable $\eta$ stands for the controlling parameter. Let $H$ be a Hilbert space and a state variable $s_{i}(\boldsymbol{x}, t ; \eta) \in H$ with $i=1,2, \cdots, r(\boldsymbol{s}) . r(\boldsymbol{s})$ is the number of state variables. The standard inner product of a state variable $s_{i}$ (between two time instances, $s_{i}\left(\boldsymbol{x}, t_{1} ; \eta\right)$ and $s_{i}\left(\boldsymbol{x}, t_{2} ; \eta\right)$,) is given by,

$$
\left(s_{i}\left(\boldsymbol{x}, t_{1} ; \eta\right), s_{i}\left(\boldsymbol{x}, t_{2} ; \eta\right)\right)_{\Omega}=\int_{\Omega} s_{i}\left(\boldsymbol{x}, t_{1} ; \eta\right) \cdot s_{i}\left(\boldsymbol{x}, t_{2} ; \eta\right) d \boldsymbol{x}
$$

The induced norm and time averaging (over the time $T$ ) of the state variable is defined as,

$$
\left\|s_{i}\right\|_{\Omega}=\sqrt{\left(s_{i}, s_{i}\right)_{\Omega}} \text { and } \bar{s}_{i}=\frac{1}{T} \int_{T} s_{i} d t=\left\langle s_{i}\right\rangle_{T} .
$$

The controlling/operating parameter $\eta$ is an important parameter of ROM. For a change in $\eta$, the ROM must predict the solution state vector $\boldsymbol{s}(\boldsymbol{x}, t ; \eta)$, which can also be performed on the fly. The flow parameters such as the Reynolds number, Mach number and angle of attack can serve as the controlling parameter. In fluid-structure interaction problems, structural parameters such as the mass ratio, non-dimensional stiffness and geometric parameters are commonly considered, depending on the objective of reduced-order modeling. The controlling parameter is also referred as control or objective function.

\subsection{The Snapshot POD}

Lumley [5] introduced the method of proper orthogonal decomposition (POD) to fluid mechanics in order to analyze coherent turbulent flow structures. The direct POD method involves, generally, a large number of 
degrees-of-freedom when applied to a CFD simulation. The method of snapshot POD, proposed by Sirovich [6], results in significant reduction of the number of degrees-of-freedom. In an arbitrary Lagrangian Eulerian (ALE) formulation for fluid-structure coupling the computational mesh is re-positioned in accordance with the moving solid boundaries. A simple numerical approach to handle the mesh is to solve the Laplace equation for the mesh deformation by retaining the mesh topology. Let the solution state vector be $\boldsymbol{s}(\boldsymbol{x}, t ; \eta)$, which includes the deforming mesh in addition to the flow state variables. The solution state vector can be written as,

$$
\boldsymbol{s}(\boldsymbol{x}, t ; \eta)=\left(\begin{array}{c}
\chi_{i}(\boldsymbol{x}, t ; \eta) \\
\rho(\boldsymbol{x}, t ; \eta) \\
v_{i}(\boldsymbol{x}, t ; \eta) \\
p(\boldsymbol{x}, t ; \eta)
\end{array}\right) \quad \text { with } \quad i=1,2,3
$$

where the variables $\rho, v_{i}$ and $p$ are the density, components of velocity vector and pressure respectively. A number of solution states or snapshots of an accurate high-fidelity (HF) CFD simulation are gathered as it advances in time. The state vector can be decomposed by using the Reynolds decomposition as,

$$
\begin{aligned}
s_{i}(\boldsymbol{x}, t ; \eta) & =\left\langle s_{i}(\boldsymbol{x} ; \eta)\right\rangle+s_{i}^{\prime}(\boldsymbol{x}, t ; \eta) \\
& =\left\langle s_{i}(\boldsymbol{x} ; \eta)\right\rangle+\sum_{j=1}^{\infty} \phi_{j}^{s_{i}}(\boldsymbol{x} ; \eta) a_{j}^{s_{i}}(t ; \eta),
\end{aligned}
$$

where $s_{i}^{\prime}(\boldsymbol{x}, t ; \eta)$ represents the unsteady part of the Reynolds decomposition. The flow solution is assumed to be statistically stationary. The unsteady part of the state vector in Eq. 4 is equivalently represented by means of the time-invariant POD space modes (topos) and space-invariant POD time modes (chronos) (Eq. 5). The solution state vector can be approximated by using a reduced number of energy dominant POD modes $\left(\phi_{j}^{s_{i}}\right)$ and corresponding time coefficients $\left(a_{j}^{s_{i}}\right)$ as shown in Eq 6 .

$$
s_{i}(\boldsymbol{x}, t ; \eta) \approx\left\langle s_{i}(\boldsymbol{x} ; \eta)\right\rangle+\sum_{j=1}^{N_{r}^{s_{i}}} \phi_{j}^{s_{i}}(\boldsymbol{x} ; \eta) a_{j}^{s_{i}}(t ; \eta) \quad t_{1} \leq t \leq t_{s n}
$$

A reduced-order solution can be obtained for the time interval of snapshots collection i.e. for $\left[t_{1}, t_{s n}\right]$, where $N_{r}^{s_{i}}$ is a reduced number of POD modes for $s_{i}$; this number can be different for each variable of the solution state vector. Here $t_{1}$ and $t_{s n}$ represent time of the first and last snapshots respectively. Let $\mathcal{R}(\eta)$ be a two point time-correlation function, given by,

$$
\boldsymbol{R}(\eta)=\left(\begin{array}{c}
\mathcal{R}^{\chi_{1}}(\eta)_{\left(t_{i} \times t_{j}\right)} \\
\vdots \\
\mathcal{R}^{\rho}(\eta)_{\left(t_{i} \times t_{j}\right)} \\
\vdots \\
\mathcal{R}^{p}(\eta)_{\left(t_{i} \times t_{j}\right)}
\end{array}\right)=\frac{1}{N_{t}}\left(\begin{array}{c}
\left(\chi_{1}\left(\boldsymbol{x}, t_{i}\right), \chi_{1}\left(\boldsymbol{x}, t_{j}\right)\right)_{\Omega} \\
\vdots \\
\left(\rho\left(\boldsymbol{x}, t_{i}\right), \rho\left(\boldsymbol{x}, t_{j}\right)\right)_{\Omega} \\
\vdots \\
\left(p\left(\boldsymbol{x}, t_{i}\right), p\left(\boldsymbol{x}, t_{j}\right)\right)_{\Omega}
\end{array}\right),
$$


where $N_{t}$ stands for the number of snapshots used to estimate the time-correlation tensor. The subscript $\left(t_{i} \times t_{j}\right)$ stands for the dimensions of each element of the correlation tensor. The correlation tensor $\mathcal{R}(\eta)$ is solved for an eigenvalue problem, as in Eq. 8

$$
\mathcal{R}^{s_{i}}(\eta) a_{j}^{s_{i}}(t ; \eta)=\lambda_{j}^{s_{i}} a_{j}^{s_{i}}(t ; \eta),
$$

where $\lambda_{j}^{s_{i}}$ are the eigenvalues. The eigenfunctions $a_{j}^{s_{i}}(t ; \eta)$ satisfy,

$$
\left(a_{j}^{s_{i}}(t ; \eta), a_{k}^{s_{i}}(t ; \eta)\right)_{T_{s n}}=\delta_{j k},
$$

where, $\delta_{j k}$ is the Kronecker delta. The POD modes are arranged in descending order of their energy content (eigenvalues), i.e $\lambda_{1}^{s_{i}} \geq \lambda_{2}^{s_{i}} \geq \ldots \ldots \ldots \ldots \ldots . . .1 \lambda_{N_{p o d}}^{s_{i}}>0$. The orthonormal POD space modes are obtained using Eq. 10, such that $\left(\phi_{j}^{s_{i}}(\boldsymbol{x} ; \eta), \phi_{k}^{s_{i}}(\boldsymbol{x} ; \eta)\right)_{\Omega}=\delta_{j k}$.

$$
\phi_{j}^{s_{i}}(\boldsymbol{x} ; \eta)=\frac{1}{\sqrt{N_{t} \lambda_{j}^{s_{i}}}}\left(s_{i}^{\prime}(\boldsymbol{x}, t ; \eta), a_{j}^{s_{i}}(t ; \eta)\right)_{T_{s n}}
$$

The corresponding POD time coefficients are given by,

$$
\begin{aligned}
a_{j}^{s_{i}}(t ; \eta) & =\left(\phi_{j}^{s_{i}}(\boldsymbol{x} ; \eta), s_{i}^{\prime}(\boldsymbol{x}, t ; \eta)\right)_{\Omega} \\
& =\sqrt{N_{t} \lambda_{j}^{s_{i}}} a_{j}^{s_{i}}(t ; \eta)
\end{aligned}
$$

Generally, the number of reduced POD modes $\left(N_{r}^{s_{i}}\right)$ is much smaller compared to the total POD modes $\left(N_{r}^{s_{i}}<<N_{\text {pod }}^{s_{i}}\right)$, containing most of the energy $E_{c}$. The relative energy of reduced system is given as,

$$
\% E c^{s_{i}}=\frac{\sum_{j=1}^{N_{r}^{s_{i}}} \lambda_{j}^{s_{i}}}{\sum_{j=1}^{N_{\text {pod }}^{s_{i}}} \lambda_{j}^{s_{i}}} \times 100 .
$$

The total energy of the system can be estimated by Eq. 13 , which also provides an approximate estimate of the energy in terms of POD time coefficient $\left(a_{j}^{s_{i}}\right)$.

$$
E^{s_{i}}(\eta)=\frac{1}{2} \int_{\Omega}\left\langle s_{i}^{\prime}(\boldsymbol{x}, t ; \eta)^{2}\right\rangle_{T_{s n}} d \boldsymbol{x} \approx \frac{1}{2} \sum_{j=1}^{N_{r}^{s_{i}}} \lambda_{j}^{s_{i}}=\frac{1}{2} \sum_{j=1}^{N_{r}^{s_{i}}}\left\langle a_{j}^{s_{i}}(t ; \eta)^{2}\right\rangle_{T_{\eta}},
$$

where $T_{s n}$ is the time interval of snapshots collection. An approximate value of total energy can be obtained for a minimum value of time $T_{\eta}$, which is the time-period of one limit cycle oscillation (LCO) of the first POD mode, $a_{1}(t ; \eta)$, for an operating parameter $\eta$. Equation 14 provides characteristic time coefficients, where $t_{a}$ is an arbitrary time in $t_{a}=\left[0,\left(t_{s n}-T_{\eta}\right)\right]$.

$$
\tilde{a}_{i}^{s_{i}}(t ; \eta)=a_{i}^{s_{i}}(t ; \eta) \quad \text { for } t \in\left[t_{a}, t_{a}+T_{\eta}\right]
$$

Equation 6 can be reframed as in terms of the characteristic POD time modes as:

$$
s_{i}(\boldsymbol{x}, t ; \eta) \approx\left\langle s_{i}(\boldsymbol{x} ; \eta)\right\rangle+\sum_{j=1}^{N_{r}^{s_{i}}} \phi_{j}^{s_{i}}(\boldsymbol{x} ; \eta) \tilde{a}_{j}^{s_{i}}(t ; \eta) \quad t \geq 0,
$$

leading to a ROM that can time march outside the snapshots' time interval $\left(\left[t_{1}, t_{s n}\right]\right)$. 


\subsection{Linear interpolation}

The time average of state vector $(\langle\boldsymbol{s}\rangle)$ and the POD modes $\left(\phi_{i}^{s_{i}}, \tilde{a}_{i}^{s_{i}}\right)$ are interpolated linearly for any change in the operating parameters such as Reynolds number, mass ratio (mass of solid/mass of displaced fluid medium) in fluid-structure interaction systems by using Eq. 16 .

$$
\Gamma(\boldsymbol{\beta} ; \eta)=\Gamma\left(\boldsymbol{\beta} ; \eta_{1}\right)+\left[\frac{\left(\Gamma\left(\boldsymbol{\beta} ; \eta_{2}\right)-\Gamma\left(\boldsymbol{\beta} ; \eta_{1}\right)\right)}{\left(\eta_{2}-\eta_{1}\right)}\right]\left(\eta-\eta_{1}\right),
$$

where $\eta_{1}, \eta_{2}$ are the values of controlling parameter for two high-fidelity reference cases. $\Gamma$ is a dummy variable for $\left\langle s_{i}\right\rangle, \phi_{j}^{s_{i}}$ and $\tilde{a}_{j}^{s_{i}}$, whereas $\beta$ is replaced by $\boldsymbol{x}$ for the state variable or space modes and by $t$ for the characteristic POD time modes. A priori the standard inner product in Eq. 17 is satisfied, so that the interpolated quantities (terms on the right hand side of Eq 15) are in minimum phase difference with $\eta_{1}$ reference case.

$$
\left(\Gamma\left(\beta ; \eta_{1}\right), \Gamma\left(\beta ; \eta_{2}\right)\right)_{\beta} \geq 0
$$

The linear interpolation can be easily extended for a linear extrapolation using the reference state solutions, in order to broaden the range of operating conditions. Furthermore, a smooth transition from an operating condition to another can be achieved by using a linear transition, as discussed in Morzynski et al. 24] and Shinde et al. [23].

\subsection{Error estimate}

An error estimate based on the quadratic energy terms is considered, which provides an upper bound of the error for a Galerkin-POD ROM [25, 26]. The normalized error $(\epsilon(t ; \eta))$ can be given by,

$$
\begin{aligned}
\epsilon(t ; \eta) & =\sum_{i=1}^{r(\boldsymbol{s})}\left[\frac{\left(E^{s_{i}}(t ; \eta)_{p o d}-E^{s_{i}}(t ; \eta)\right)}{\sigma_{i}{ }^{2}(\eta)}\right]^{2} \\
& =\sum_{i=1}^{r(\boldsymbol{s})}\left[\frac{\left(\sum_{j=1}^{N_{\text {pod }}} \tilde{a}_{j}^{s_{i}}(t ; \eta)_{\text {pod }}^{2}-\sum_{j=1}^{N_{r}} \tilde{a}_{j}^{s_{i}}(t ; \eta)^{2}\right)}{\sigma_{i}{ }^{2}(\eta)}\right]^{2},
\end{aligned}
$$

where the subscript pod stands for the energy of POD reconstructed solution. The variance is computed using HF model as,

$$
\sigma_{i}^{2}(\eta)=\int_{\Omega}\left\langle s_{i}^{\prime}(\boldsymbol{x}, t ; \eta)_{h f}^{2}\right\rangle_{T} d \boldsymbol{x}
$$

\section{Results and discussion}

At first, we present a test case of vortex-induced vibration of cylinder at Reynolds number based on cylinder diameter $R e_{D}=100$. The computations are performed in two-dimensional configuration for various structural-to-fluid mass ratios, and a ROM is constructed for the predictions at off-reference values of the mass ratio. Secondly, a test case of panel flutter induced by shock wave boundary layer interaction is 
considered in order to demonstrate the applicability of the proposed method for three-dimensional and highly non-linear fluid-structure interaction problems. For this case, we consider an existing high-fidelity simulation database from Shinde et al. [27, where an incoming laminar boundary layer at Mach 2 and Reynolds number base on panel length $R e_{a}=50000$ interacts with an incident shock at the shock angle of $35^{\circ}$ and shock strength of 1.8 over a flexible panel. The study [27] comprises effect of several structural parameters on the panel flutter, however we consider only the effect of panel thickness, and construct a ROM solution for the flutter response and wall pressure of the panel.

\subsection{Vortex-induced vibration of a cylinder at $R e_{D}=100$ for various mass ratios}

The two-dimensional vortex-induced vibration (VIV) of a circular is at Reynolds number $\operatorname{Re}_{D}=100$ based on the cylinder diameter $D$, streamwise inflow velocity $u_{\infty}$, fluid density $\rho$ and fluid dynamic viscosity $\mu$. The structural parameters include the mass ratio $m^{*}=m /\left(\rho D^{2}\right)$, cylinder natural frequency $f_{n}$ (in vacuum) and damping coefficient $\zeta$, where $m$ represents the cylinder mass per unit length. Figure 1 shows the computational domain $(\chi)$ and instantaneous streamwise velocity field $(u(\chi, t ; \eta))$, where the control parameter $\eta=m^{*}$. The simulations are performed by using standard Dirichlet and far field boundary conditions, leading to the mean flow in $+X$ direction. The cylinder surface is a no-slip adiabatic wall.

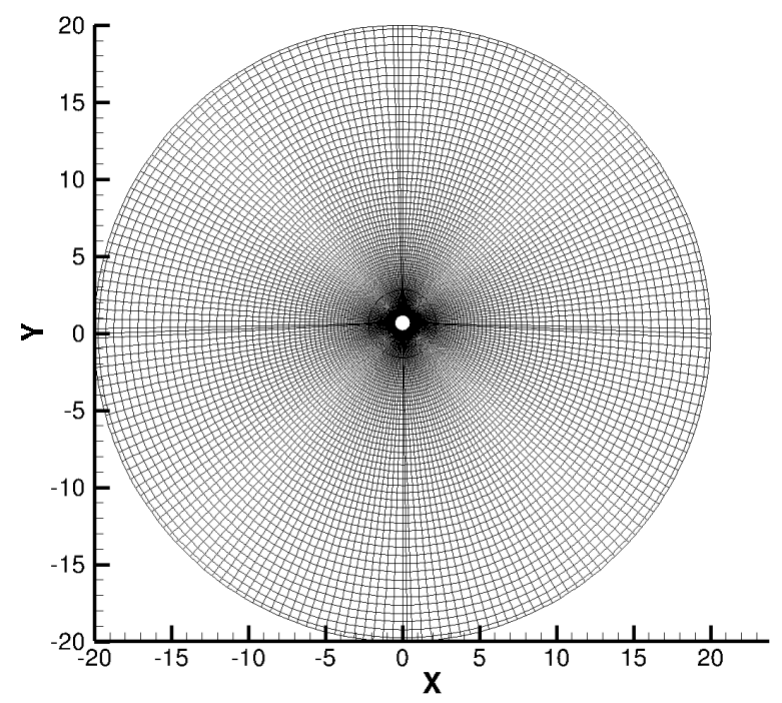

(a) Geometry and mesh

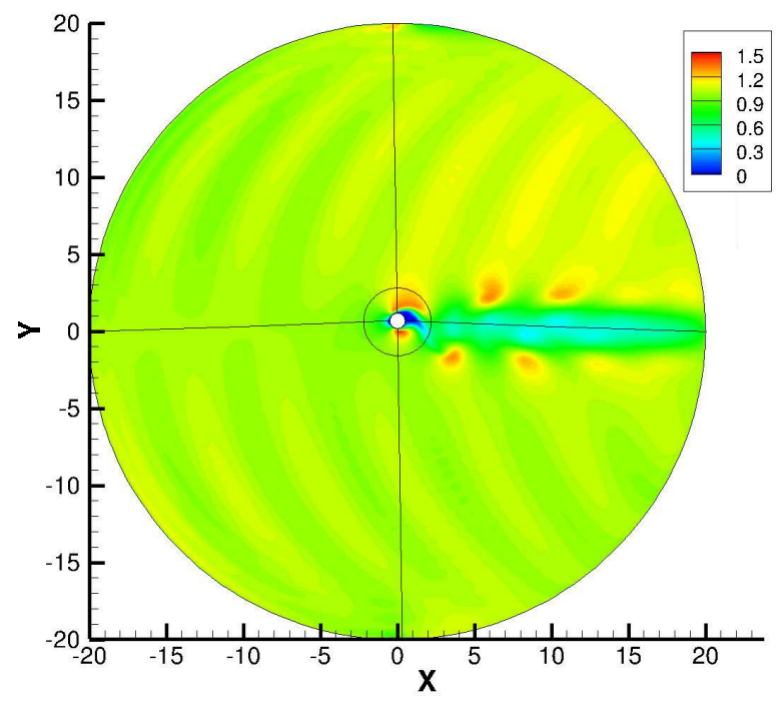

(b) Instantaneous streamwise velocity

Figure 1: Computational domain and instantaneous flow field at $R e=100$ and mass ratio $m^{*}=2.50$

\subsubsection{The flow governing equations}

A compressible flow solver [28, 29] with an appropriate preconditioning scheme for the flow incompressibility is used for the HF CFD simulations. The full Navier-Stokes Equations (NSE) govern the fluid flow, 
and are described in Eq. 20 through Eq. 22 .

$$
\frac{\partial}{\partial t}(\boldsymbol{w})+\frac{\partial}{\partial x}\left(\boldsymbol{f}-\boldsymbol{f}_{\nu}\right)+\frac{\partial}{\partial y}\left(\boldsymbol{g}-\boldsymbol{g}_{\nu}\right)=0,
$$

where

$$
\begin{aligned}
\boldsymbol{w}= & \left(\begin{array}{c}
\rho \\
\rho u \\
\rho v \\
\rho E
\end{array}\right), \boldsymbol{f}=\left(\begin{array}{c}
\rho u \\
\rho u^{2}+p \\
\rho u v \\
u(\rho E+p)
\end{array}\right), \boldsymbol{g}=\left(\begin{array}{c}
\rho v \\
\rho v u \\
\rho v^{2}+p \\
v(\rho E+p)
\end{array}\right) \\
\boldsymbol{f}_{\nu}= & \left(\begin{array}{c}
0 \\
\tau_{x x} \\
\tau_{x y} \\
{[\boldsymbol{\tau}, \mathbf{v}]_{x}-q_{x}}
\end{array}\right), \boldsymbol{g}_{\nu}=\left(\begin{array}{c}
0 \\
\tau_{y x} \\
\tau_{y y} \\
{[\boldsymbol{\tau}, \mathbf{v}]_{y}-q_{y}}
\end{array}\right) .
\end{aligned}
$$

Here $\boldsymbol{w}$ is the state vector. $\boldsymbol{f}, \boldsymbol{g}$ are the convective fluxes, while $\boldsymbol{f}_{\nu}, \boldsymbol{g}_{\nu}$ are the viscous fluxes. The flow velocity components in $x$ and $y$ directions are denoted by $u$ and $v$ respectively. $p$ stands for the pressure, while $E$ represents the specific energy density. The components of shear stress tensor $\tau$ in the viscous fluxes are given by Eq. 21

$$
\begin{aligned}
\tau_{x x} & =\frac{2}{3} \mu\left(2 \frac{\partial u}{\partial x}-\frac{\partial v}{\partial y}\right), \tau_{y y}=\frac{2}{3} \mu\left(-\frac{\partial u}{\partial x}+2 \frac{\partial v}{\partial y}\right) \\
\tau_{x y} & =\tau_{y x}=\mu\left(\frac{\partial u}{\partial y}+\frac{\partial v}{\partial x}\right)
\end{aligned}
$$

The heat flux is calculated using Fourier's law as,

$$
q_{x}=-k_{\theta} \frac{\partial \theta}{\partial x}, q_{y}=-k_{\theta} \frac{\partial \theta}{\partial y} \quad \text { with } k_{\theta}=\mu C_{p} / \operatorname{Pr},
$$

where $k_{\theta}, \theta$ are the thermal conductivity, temperature respectively. The Prandtl number $(P r)$ is taken 0.72 (for air).

The second order fully implicit LU-SGS (Lower-Upper Symmetric Gauss-Seidel) backward A-stable scheme is used for solving the discretized equations, as discussed in Vos et al. [30. The space derivatives are discretized by using a forth order central finite volume scheme in a skew-symmetric form. For the incompressibility, the preconditioning method proposed in Turkel et al. 31 is used for the flows with low Mach numbers.

\subsubsection{Fluid-structure coupling}

The cylinder's motion is modeled as the mass on a spring system, as shown in Fig. 2, where the cylinder oscillates only in the lift direction ( $Y$ direction). The equation of cylinder motion can be written as: 


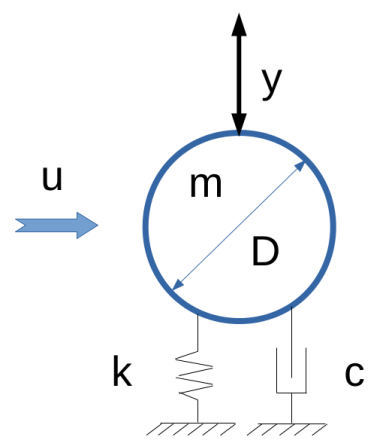

Figure 2: Coupling between fluid and the cylinder

$$
m \frac{d^{2} y}{d t^{2}}+c \frac{d y}{d t}+k y=F_{y}
$$

where $y$ is the cylinder displacement in the lift direction. $c$ and $k$ denote the structural damping and stiffness coefficients respectively. The fluid force $F_{y}$, per unit length of the cylinder, drives the motion of cylinder.

The second order differential equation is solved using Hilber-Hughes-Taylor method (HHT) Newmark algorithm [32]. An arbitrary Lagrangian Eulerian (ALE) method is used to simulate the fluid-structure coupling [28], where the displacement $(y)$ and velocity $\left(\frac{d y}{d t}\right)$ of the cylinder are used to deform the mesh and update the flow velocities respectively.

The non-dimensional flow and structure parameters are listed in Table 1 , where $u^{*}=u /\left(f_{n} D\right)$ is the non-dimensional reduced velocity. The structural non-dimensional frequency $f_{n}^{*}=f_{n} D / u_{\infty}$, leading to $f_{n}^{*}=1 / u^{*}$ at the far field. The damping coefficient $\zeta$ and natural frequency $f_{n}$ are respectively expressed as:

$$
\zeta=\frac{c}{2 \sqrt{k m}} \quad \text { and } \quad f_{n}=\frac{1}{2 \pi} \sqrt{\frac{k}{m}} .
$$

The high value of the damping ratio is selected in order to control the amplitude of the cylinder oscillations. The flow past a cylinder for Reynolds number $R e_{D}=100$ results in a Strouhal number, $S t=f_{s h} D / u_{\infty}$, of $S t \approx 0.17$, where $f_{s h}$ is the vortex shedding frequency. Thus, the cylinder frequency $f_{n}^{*}=0.17$ leads to the VIV.

\begin{tabular}{cccc}
$R e_{D}$ & $u^{*}$ & $f_{n}^{*}$ & $\zeta(\%)$ \\
\hline 100 & 5.88 & 0.17 & 20 \\
\hline
\end{tabular}

Table 1: Simulation parameters

As noted before, the mass ratio $m^{*}$ is considered as the controlling parameter $\left(\eta=m^{*}\right)$ for the VIV. The 
High-fidelity simulations are performed for a range of mass ratio values, such that the oscillation amplitudes of the cylinder vary approximately between $y \approx 0$ and $y \approx 1 D$. Table 2 shows the reference cases simulated using different mass ratio values and the corresponding response of the cylinder in terms of a root mean squared displacement $y_{r m s}^{*}=y_{r m s} / D$.

\begin{tabular}{ccccccccccc}
\hline$m^{*}$ & 1.5 & 1.75 & 2 & 2.5 & 3 & 3.5 & 5 & 7 & 10 & 20 \\
\hline$y_{r m s}^{*}$ & 0.69 & 0.64 & 0.59 & 0.51 & 0.44 & 0.38 & 0.25 & 0.21 & 0.10 & 0.04 \\
\hline
\end{tabular}

Table 2: Reference case simulations

\subsection{3. $P O D$ analysis}

The solution state vector $\boldsymbol{s}(\boldsymbol{x}, t ; \eta)$ of Eq. 3 becomes

$$
\boldsymbol{s}\left(\boldsymbol{x}, t ; m^{*}\right)=\left(\begin{array}{c}
y\left(\boldsymbol{x}, t ; m^{*}\right) \\
u\left(\boldsymbol{x}, t ; m^{*}\right) \\
v\left(\boldsymbol{x}, t ; m^{*}\right) \\
p\left(\boldsymbol{x}, t ; m^{*}\right)
\end{array}\right)
$$

for the VIV of cylinder case study. A set of snapshots of the solution vector are recorded for the snapshot POD analysis, where a total of 576 snapshots are collected using a non-dimensional time step of $\Delta t=0.05$. A time-correlation tensor is formed by using the unsteady part $\left(\boldsymbol{s}^{\prime}\right)$ of the state vector, which is then solved for the eigenvalue problem as per the procedure detailed in Sec. 2. The $\%$ energy $\left(\lambda_{i} / \sum_{i} \lambda_{i} \times 100\right)$ associated with the POD modes of the different state variables is shown in Fig. 3 for the mass ratio of $m^{*}=2.50$. The

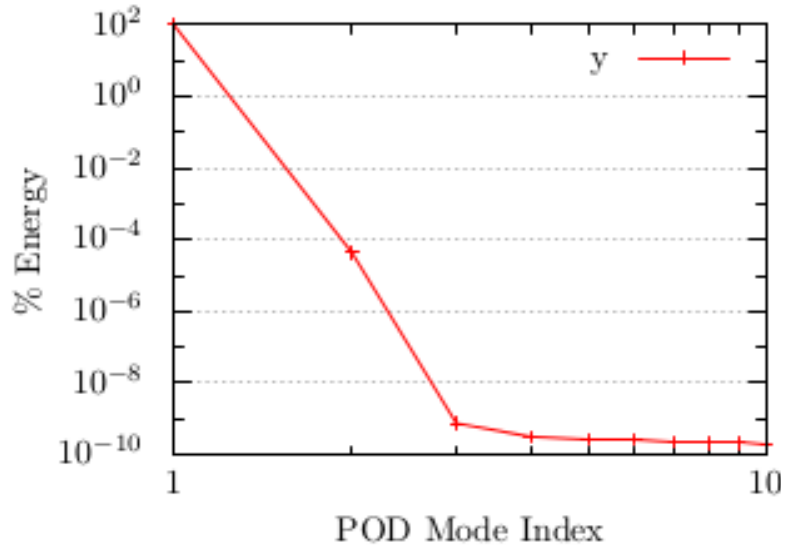

(a) mesh deformation $(y)$

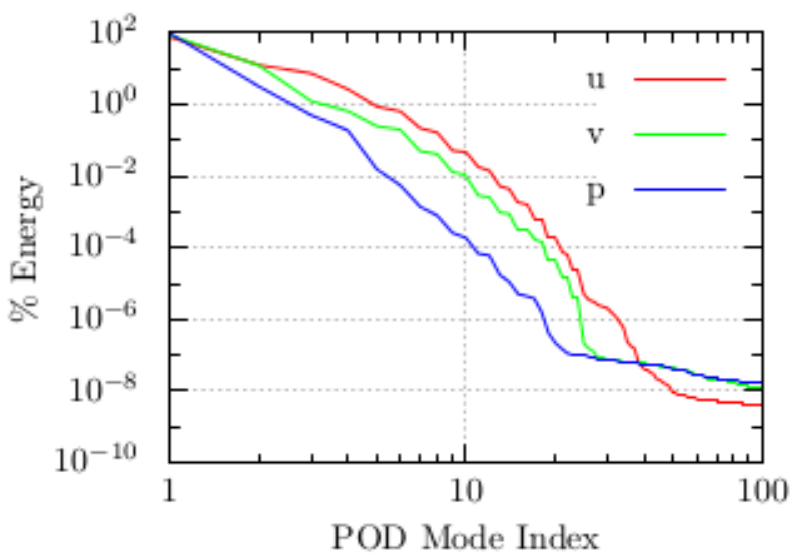

(b) flow variables $(u, v$ and $p)$

Figure 3: $\%$ energy associated with the POD modes at $R e_{D}=100, m^{*}=2.50$ 
POD modal energies are arranged in decreasing order for all variables The energy associated with the first POD mode of mesh deformation is $\approx 100 \%$ (Fig. 3 a). Thus, only one POD mode $\left(N_{r}^{y}=1\right)$ is sufficient for the reconstruction of mesh movement. On the other hand, first 10 POD modes of the velocity $(u, v)$ and pressure comprise $\approx 99 \%$ of energy, thus only 10 out of 576 modes are retained for the ROM. Furthermore, the corresponding temporal modes exhibit stable limit cycle oscillations, ensuring the periodicity required in order to extract the characteristic time modes of Eq. 14. The pair of first POD modes for the mesh

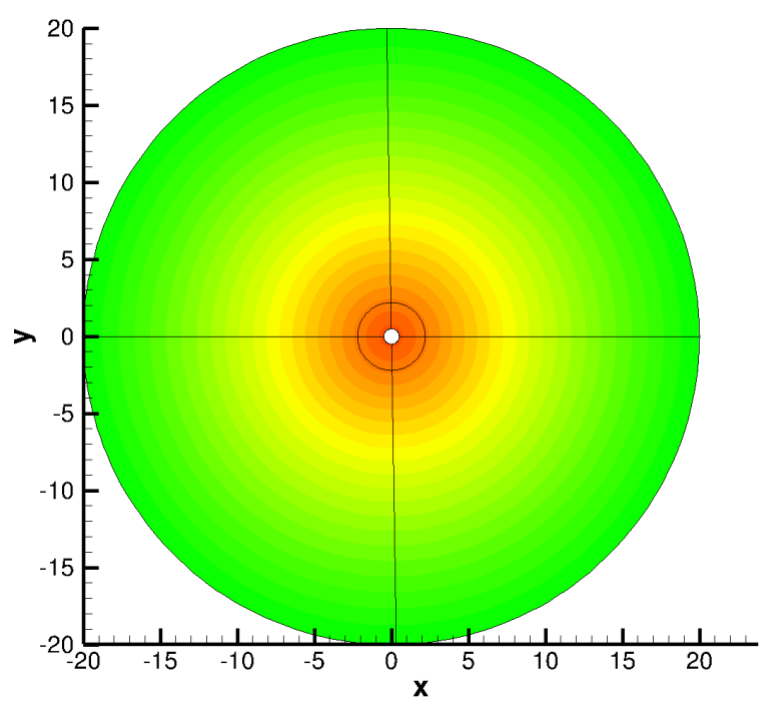

(a) Space mode $\left(\phi_{1}^{y}\left(\boldsymbol{x} ; m^{*}\right)\right)$

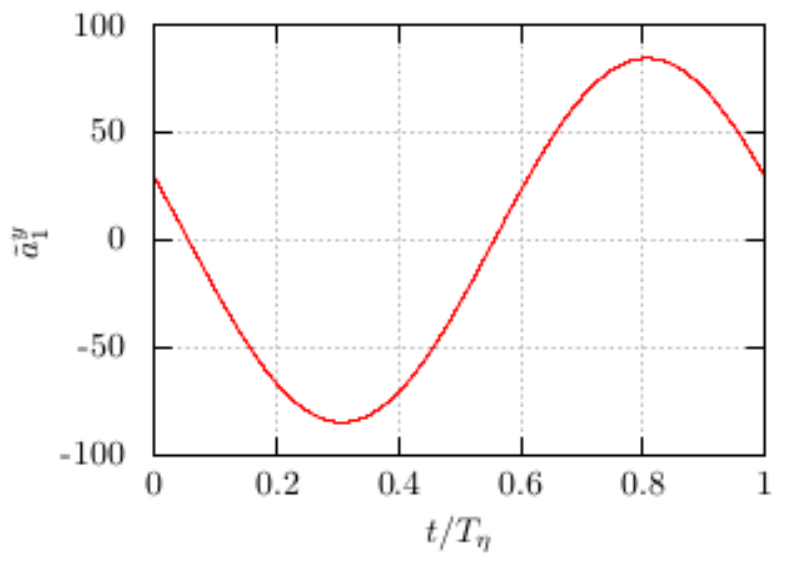

(b) Time mode $\left(a_{1}^{y}\left(t ; m^{*}\right)\right)$

Figure 4: The pair of first POD modes for mesh deformation in y direction for $\operatorname{Re}_{D}=100$ and $m^{*}=2.50$.

deformation is shown in Fig. 4, displaying the space mode $\left(\phi_{1}^{y}\left(\boldsymbol{x} ; m^{*}\right)\right)$ in Fig. 4(a) and corresponding characteristic temporal mode $\left(\tilde{a}_{1}^{y}\left(t ; m^{*}\right)\right)$ in Fig. $4(\mathrm{~b})$, where the period of LCO is $T_{m^{*}}=5.79622$.

\subsubsection{ROM solution}

The vortex-induced vibration in a circular cylinder is extensively studied in the past [33]. There exists a critical minimum value of the mass ratio, as discussed in Williamson and Govardhan [33, which is $m^{*} \approx$ 0.424 this value of critical minimum mass ratio changes with other system parameters. In order to obtain the desired higher amplitudes of cylinder oscillations, the very high value $(20 \%)$ of the damping ratio is used, varying the mass-damping parameter $\left(m \delta / \rho D^{2}\right.$ [34, where $\left.\delta \approx 2 \pi \zeta\right)$ from 0.2 to 4 . The physics of the problem at small and very small mass-damping parameter is studied in Khalak and Williamson [35] and Khalak and Williamson [36].

\footnotetext{
${ }^{1}$ In general, the mass ratio is defined as the structural mass to displaced fluid mass: $m^{*}=4 m /\left(\rho \pi D^{2}\right)$. Then the minimum critical value is $\approx 0.54$.
} 
The reduced number of POD modes $\left(N_{r}^{s_{i}}\right)$ with high energy content are selected to form the POD subspace. There are two main problems while constructing the ROM for this FSI system: first, the traditional Galerkin projection of the Navier-Stokes equations on the POD time invariant modes (or space modes) when the computation domain is time dependent, which is discussed in a great detail by Tadmor et al. 37. Second, the validity of a POD-based ROM is generally limited to a small range of controlling parameter, since the POD reduced basis non-linearly depends on the controlling parameter. Bui-Thanh et al. 38 proposed a gappy POD procedure to construct the off-reference ROM solutions, thereby dealing with the change of controlling parameters. The method uses the POD coupled with an interpolation method, which avoids the Galerkin projection of the governing equations. In Lieu et al. [39] a ROM for a complete aircraft is formulated based on a Mach-adaptation strategy, where the angle between POD subspaces is interpolated in order to deal with the changes in controlling parameter. The interpolation of the reduced basis for the change in controlling parameter is performed in a tangent space to a Grassmann manifold in Amsallem and Farhat [40] and further on matrix manifolds in Amsallem and Farhat [41]. In the present work, the periodic reconstruction of the POD time modes as per Eq. 14 is used to circumvent the Galerkin projection; and the solution, including mesh deformation, is readily built using Eq. 15. A direct linear interpolation of the POD space as well as time modes is performed in order to predict an off-reference solution state by using the pre-simulated reference cases.

The linear interpolation of the energy dominant $\left(N_{r}^{s_{i}}\right)$ POD modes is performed for any value of the mass ratio in the range of reference cases listed in Table 2 A comparison between the interpolated POD modes and high-fidelity POD modes for an off-reference case (mass ratio $m^{*}=2.75$ ) is shown in Fig. 5. The figure compares first three time-invariant POD modes (topos) of the streamwise velocity (u). Figure 5 (a), (c) and (e) show the first three energy dominant POD modes $\left(\phi_{1}^{u}, \phi_{2}^{u}\right.$ and $\left.\phi_{3}^{u}\right)$ respectively, which are obtained by using the HF simulation. In comparison, the POD modes in Fig. 5(b), (d) and (f) are the corresponding linearly interpolated POD modes, which are obtained by using the reference state POD reduced sub-spaces at $m^{*}=2.50$ and $m^{*}=3.00$. A similar configuration at Reynolds number $R e=1690$ is treated in Liberge and Hamdouni [42], where a non-linear FSI ROM based on a multiphase formulation of the Navier-Stokes equations and its extension in the solid domain is presented; in their study, Reynolds number is considered as a controlling parameter.

In addition to the interpolation of the POD space modes, the temporal coefficients are also linearly interpolated in phase space. The interpolation of the first three temporal coefficients in phase space is shown in Fig. 6. The interpolated chronos (in blue colour, Fig. 6) coincide with the high-fidelity modes beneath (in green colour, Fig 6), indicating a high accuracy of the presented FSI-ROM.

Figure 7 shows a flow chart of the operating principle of the presented reduced-order approach. Once the reference high-fidelity simulations are performed, one can build the reference POD basis. The POD reduced bases are formed separately for each state variable, including the mesh deformation. For a given 


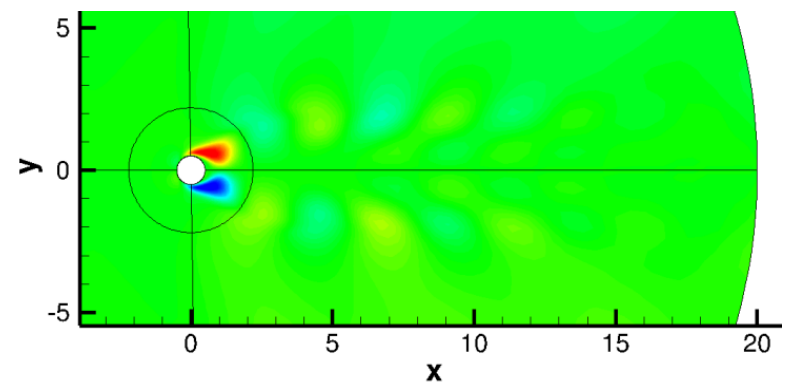

(a) $\phi_{1}^{u}\left(\boldsymbol{x} ; m^{*}\right)$ (high-fidelity mode)

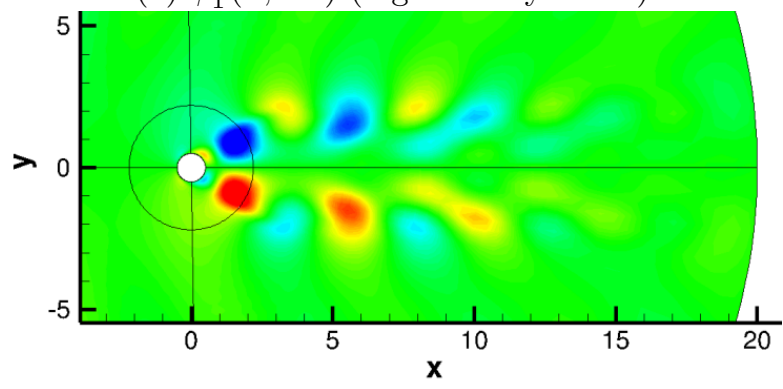

(c) $\phi_{2}^{u}\left(\boldsymbol{x} ; m^{*}\right)$ (high-fidelity mode)

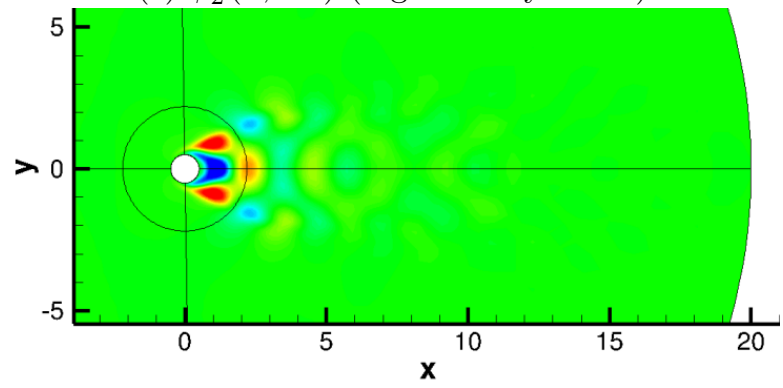

(e) $\phi_{3}^{u}\left(\boldsymbol{x} ; m^{*}\right)$ (high-fidelity mode)

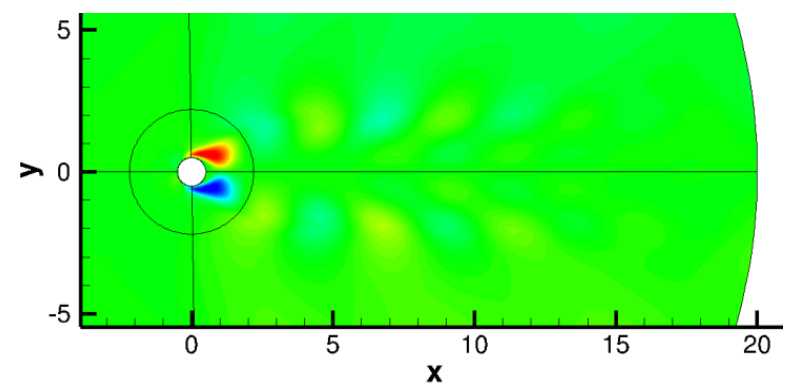

(b) $\phi_{1}^{u}\left(\boldsymbol{x} ; m^{*}\right)$ (interpolated mode)

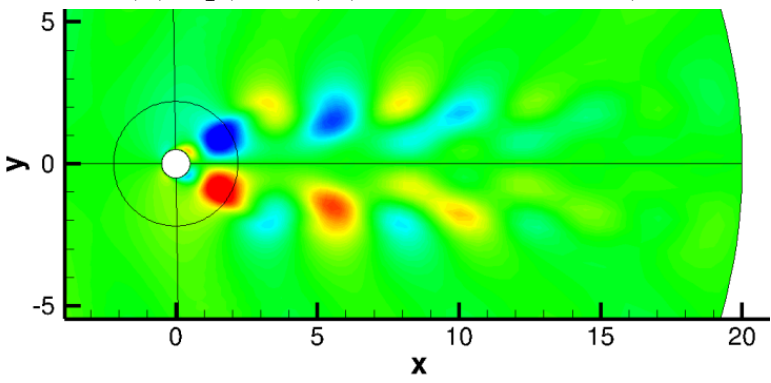

(d) $\phi_{2}^{u}\left(\boldsymbol{x} ; m^{*}\right)$ (interpolated mode)

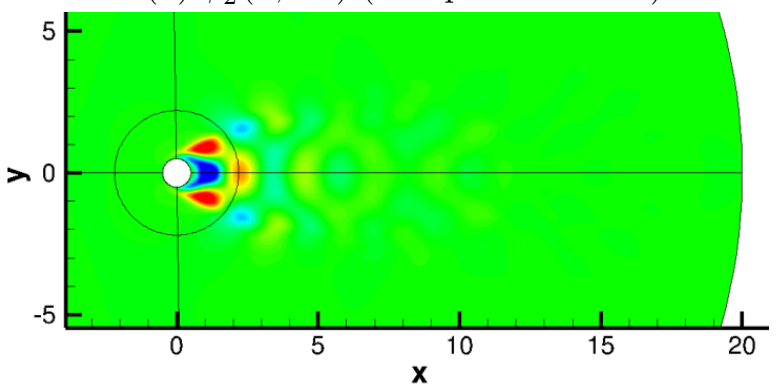

(f) $\phi_{3}^{u}\left(\boldsymbol{x} ; m^{*}\right)$ (interpolated mode)

Figure 5: Comparison of the POD modes (HF simulations) with interpolated modes at $R e=100, m^{*}=2.75$ 


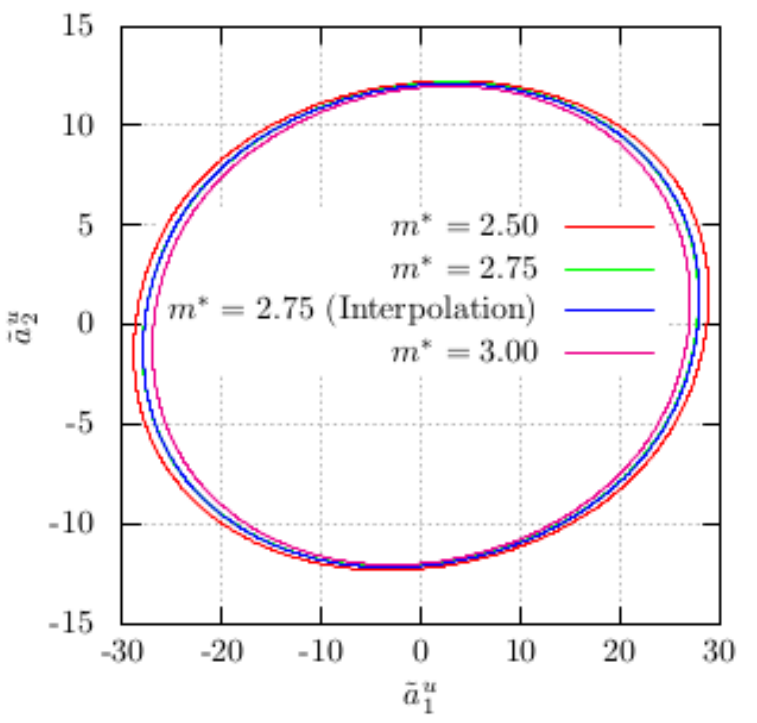

(a) $\tilde{a}_{1}^{u}\left(t ; m^{*}\right)$ Vs. $\tilde{a}_{2}^{u}\left(t ; m^{*}\right)$

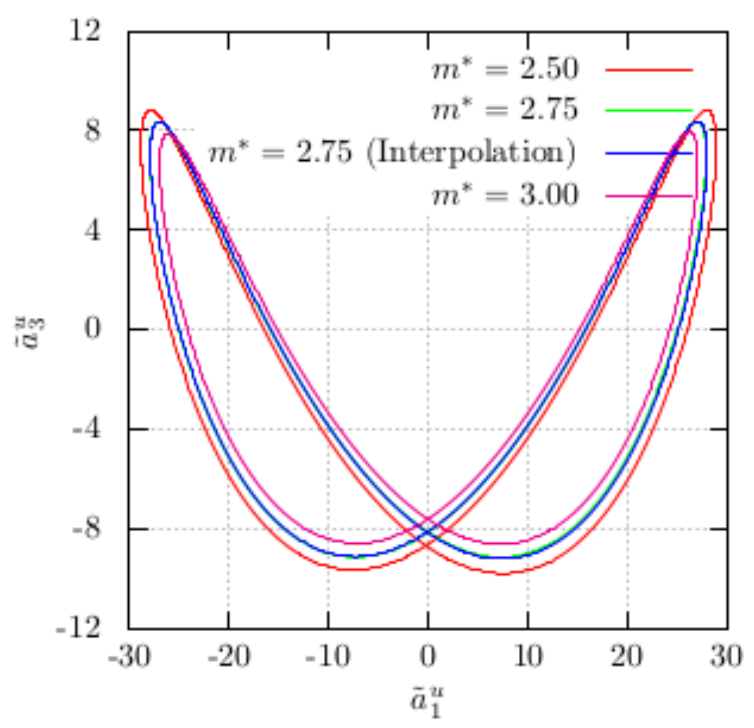

(b) $\tilde{a}_{1}^{u}\left(t ; m^{*}\right)$ Vs. $\tilde{a}_{3}^{u}\left(t ; m^{*}\right)$

Figure 6: Comparison of POD temporal coefficients (HF simulations) with interpolated modes at $R e=100, m^{*}=2.75$

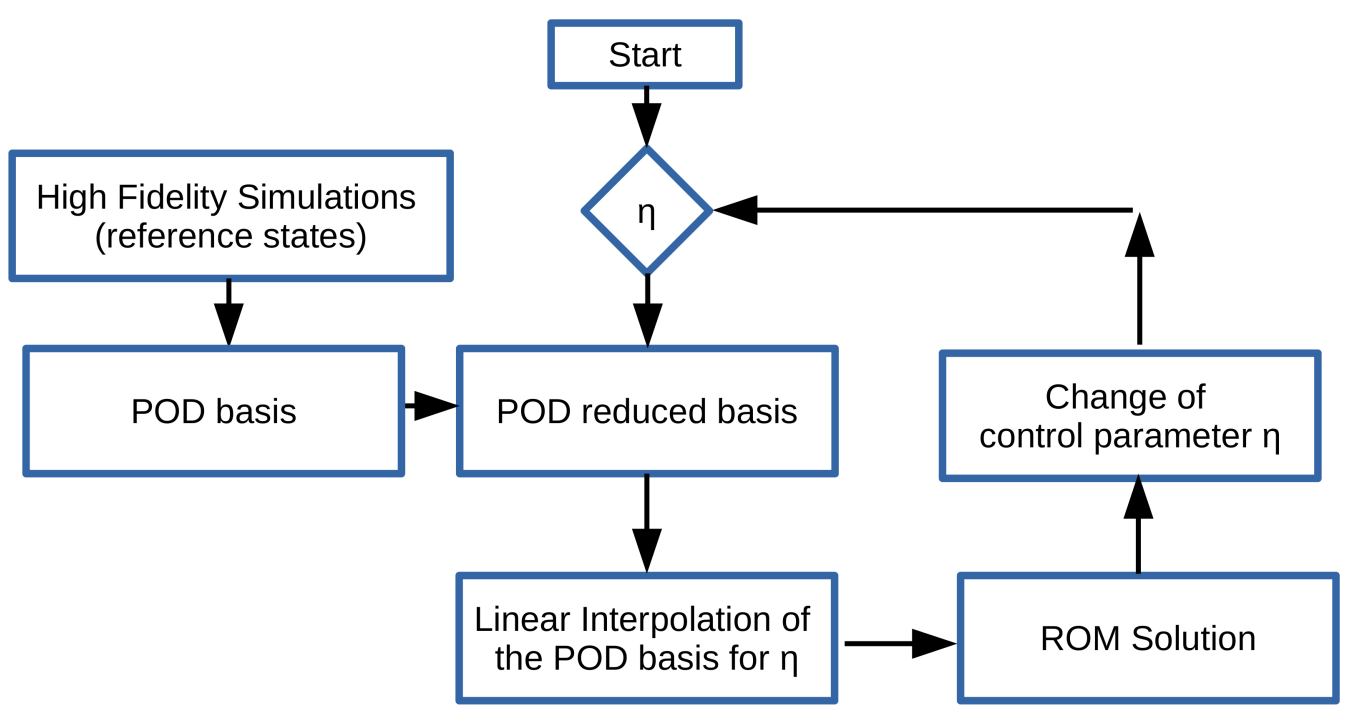

Figure 7: Working principle of the presented ROM 
value of a controlling parameter, nearest reference bases are selected for linear interpolation, as detailed in Sec. 2.2. A reduced representation of the solution state vector, comprising the mesh deformation, can be readily obtained by using the interpolated bases. A continuous update of the solution, following changes in the controlling parameter, can be obtained by using the method described in Shinde et al. 23].

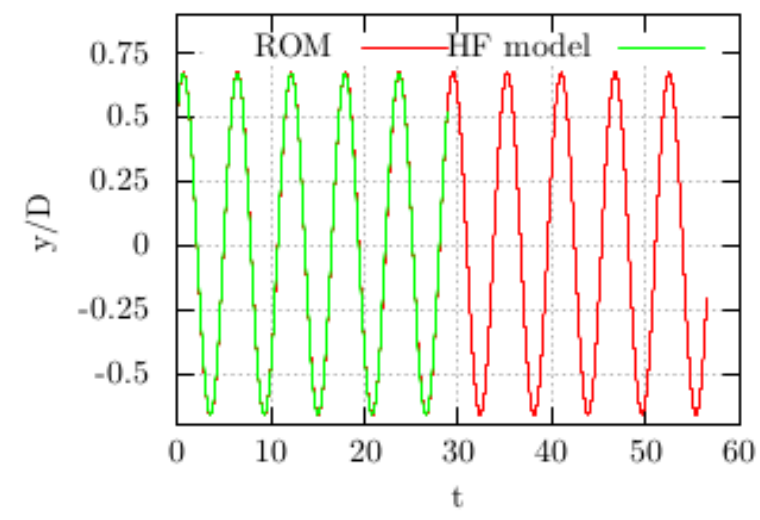

(a) Cylinder response at $m^{*}=2.75$

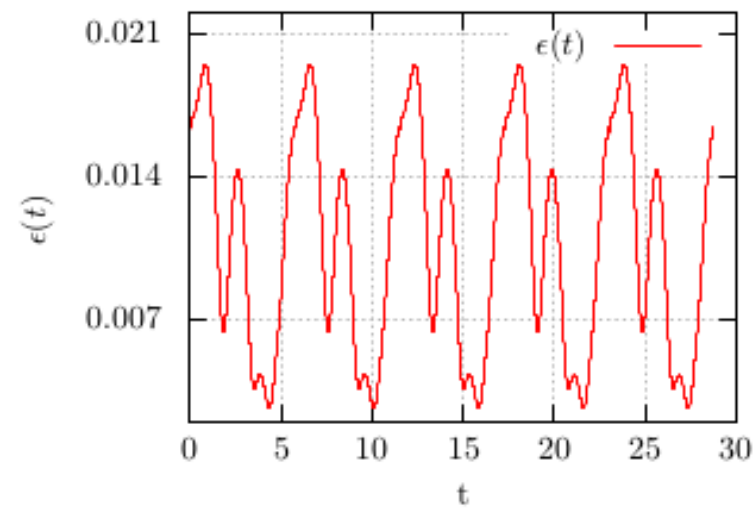

(b) Error $\epsilon(t)$

Figure 8: Comparison between the ROM and HF CFD solutions at $\operatorname{Re}_{D}=100, m^{*}=2.75$

Figure 8(b) shows the normalized energy based error $(\epsilon(t))$ as defined in Eq. 8, limiting the errors to $2 \%$. The ROM solution is obtained within $\approx 20 s$ using a single processor by following the presented ROM model. On the contrary, the corresponding HF CFD simulation takes more than 24 hours on 8 processors to provide the solution. Figure 9 shows the instantaneous streamwise velocity curve compared between the

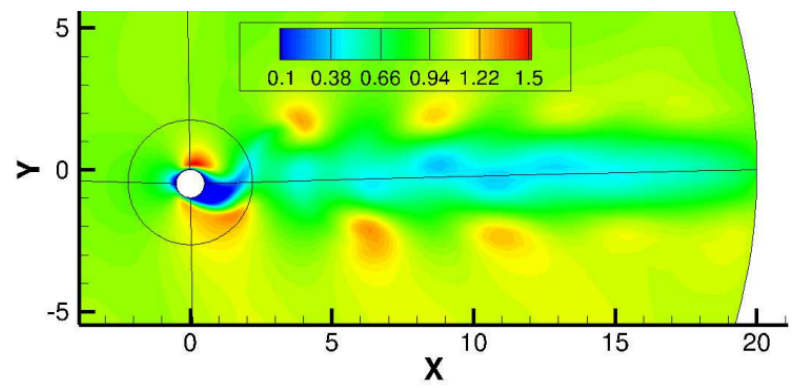

(a) $u$ at $t=10$ (high-fidelity)

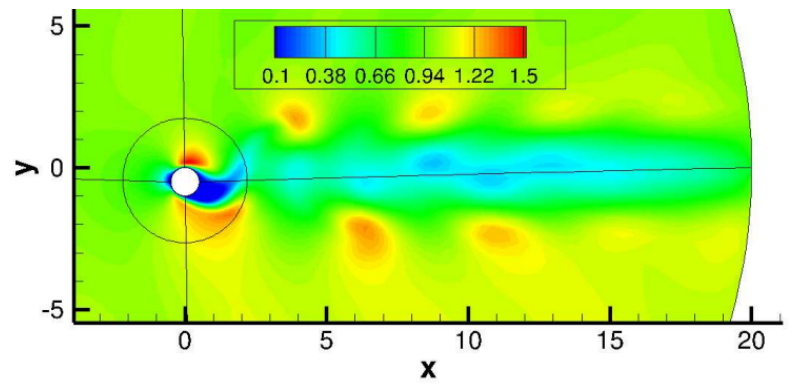

(b) $u$ at $t=10(\mathrm{ROM})$

Figure 9: Comparison of streamwise velocity $(u)$ at $t=10$ for $m^{*}=2.75$

HF CFD model and FSI-ROM, for the same simulation time of $t=10$, starting from an arbitrary stationary state.

Similar to most of the existing POD-based ROM, this method is also a posteriori formulation, which implies that the high-fidelity simulations need to be performed in order to obtain the reference cases. The 
formulation is based on the assumption of statistical stationarity of the coupled fluid-structure system. Although this test case is a periodic flow, the method is not restricted to periodic flows but the stationary flows. This aspect of the ROM is already discussed in the previous work [23, 43] based on the periodicity of the POD time coefficients in a stationary flow. For instance, Rempfer and Fasel [4] have presented the POD time coefficients of the vorticity for a flat plate boundary layer turbulent flow. Although the flow is not periodic, the POD time coefficients are periodic. A large difference between two reference states may lead to a higher interpolation error, due to the linear interpolation as well as due to the order/rank change of the POD modes.

\subsection{A shock wave boundary layer induced panel flutter}

The flow configuration of a shock wave boundary layer induced panel flutter consists of an oblique incident shock at the shock angle of $35^{\circ}$ and shock strength of $p_{3} / p_{1}=1.8$. The schematic of flow configuration is shown in Fig. 10(a). The incident shock interacts with a laminar boundary layer that evolves from a Blasius profile, where the inflow boundary thickness is $\delta_{i n} / l=0.0083$, inflow Mach number $M_{\infty}=2.0$ and Reynolds number based on panel length $R e_{l}=50000$. The shock impinges at the mid-chord location of the panel, where the panel length $(l)$ to width $(b)$ ratio is $b / l=1$. The pressure inside the cavity that is beneath the flexible panel is $p_{c} / p_{1}=1.4$. Shinde et al. [27] investigated the panel flutter for various structural parameters that include: panel aspect ratio $b / l$, panel thickness $h^{*}=h / l$, flutter parameter and mass ratio. Figure 10(b) shows a three-dimensional flow field in terms of the $Q$ criterion isosurface colored with velocity magnitude, shadowgraph (Laplacian of the density) and panel deflection contours, exhibiting a transitional shock wave boundary layer interaction (SWBLI) over a flexible panel, for a set of structural parameters. The thick lined square on the bottom surface indicates the panel edges (Fig. $10 \mathrm{~b}$ ).

The simulations in Shinde et al. 27] were performed by using a well validated aeroelastic solver [45, 46, 47, 48. A 2nd order implicit time marching scheme alongside a $6 t h$ order compact difference scheme was used for solving Navier-Stokes equation in a full three-dimensional curvilinear formulation [49, 50]. The structural dynamics of the flexible panel was modeled by using von-Karman plate theory. The spatial derivatives in the structural equations were solved using standard $2 n d$ order central finite difference scheme, whereas the equations were time-marched by using a second order unconditionally stable Newmarks $\beta$ algorithm. Further numerical details on the fluid-structure coupling are elaborated in Gordnier and Visbal [51.

We consider the effect of panel thickness $h^{*}$ on panel flutter in order to construct the proposed Galerkinfree POD-ROM, thus the controlling parameter of ROM is the panel thickness $\left(\eta=h^{*}\right)$. Furthermore, we consider only a computational plane of the geometry for the ROM that includes the panel, and a solution state vector comprising only the panel deflection and wall pressure, which are important variables for panel flutter. Table 3 lists two reference cases and corresponding flutter responses in terms of the mean $\left(y_{m}\right)$ and standard deviation $\left(y_{s d}\right)$ of panel deflection at a $3 / 4$ chord length of panel along the mid-span location. $z$ 


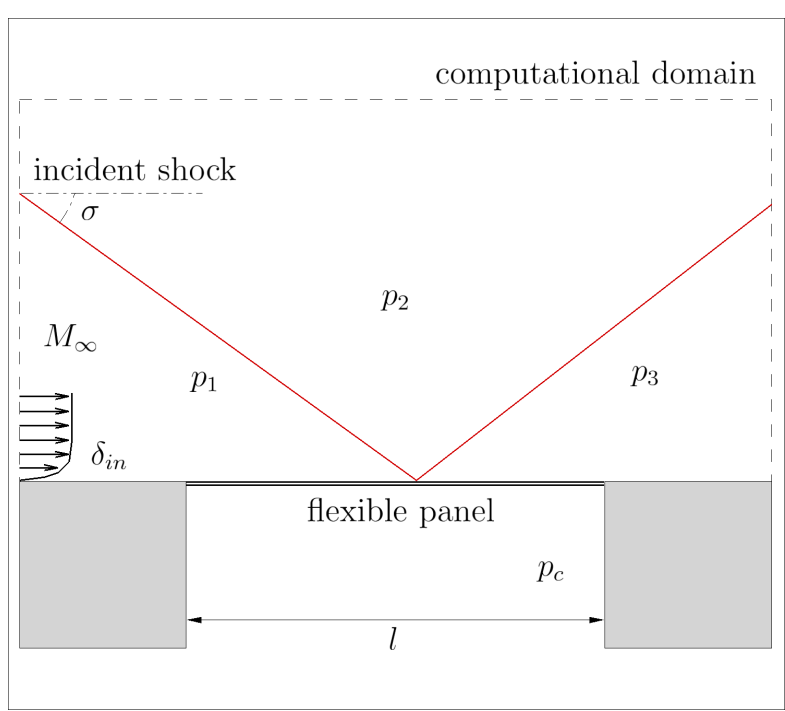

(a)

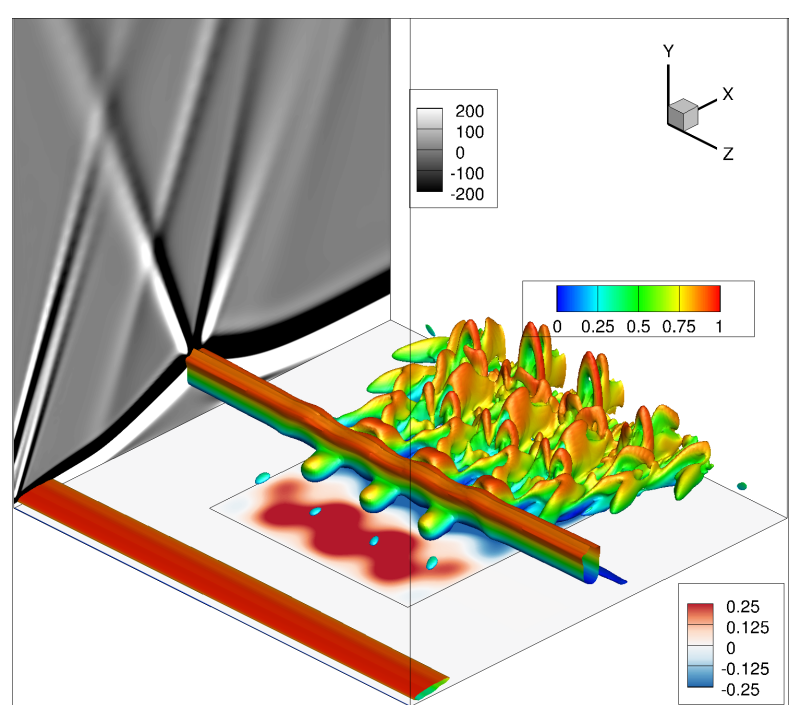

(b)

Figure 10: Flow configuration. (a) schematic of the computational domain (b) an instantaneous three-dimensional fluidstructure interaction state. $y$ axis is scaled by factor 4 for clarity.

axis represents the spanwise direction (refer Fig. $10 \mathrm{~b}$ ), while $x$ and $y$ are the streamwise and wall normal directions respectively.

\begin{tabular}{cccccc}
\hline$h^{*}$ & 0.002 & 0.004 & 0.008 & 0.016 & 0.032 \\
\hline$y_{m} / h$ & -1.6131 & -1.2864 & -1.0001 & -0.7240 & -0.4179 \\
\hline$y_{s d} / h$ & 0.1372 & 0.0974 & 0.05338 & 0.02964 & 0.0122 \\
\hline
\end{tabular}

Table 3: Reference case simulations

\subsubsection{POD analysis}

The solution state vector of Eq. 3 comprises the panel deflection $y$ and the wall pressure $p_{w}$, given as:

$$
\boldsymbol{s}\left(\boldsymbol{x}, t ; h^{*}\right)=\left(\begin{array}{c}
y\left(\boldsymbol{x}, t ; h^{*}\right) \\
p\left(\boldsymbol{x}, t ; h^{*}\right)
\end{array}\right) .
$$

The simulations in Shinde et al. 27] were performed for sufficiently long non-dimensional time, ensuring the statistical stationarity of the flutter response. A total of 1000 snapshots are collected using a time interval of $\Delta t=0.01$ in order to perform the snapshot POD analysis as detailed in Sec. 2. The snapshot POD is performed for both the reference cases, while the results for panel thickness $h / l=0.008$ are presented in this section.

The POD modal energy curves in Fig. 11(a) show the percentage energy contained in each mode for the panel deflection and wall pressure. The snapshot procedure leads to a total of 1000 POD modes for 


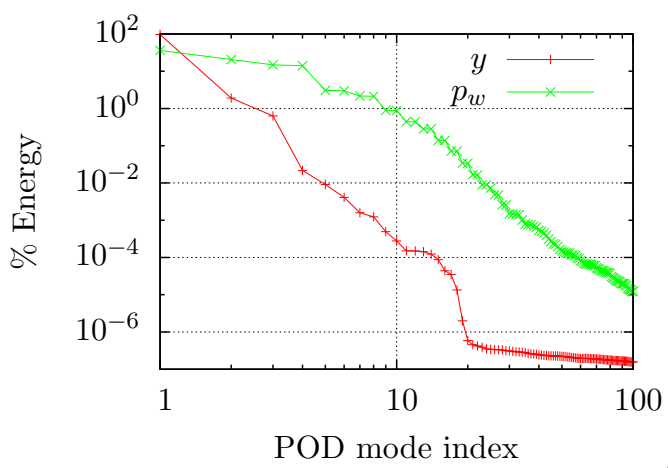

(a) POD modal energy

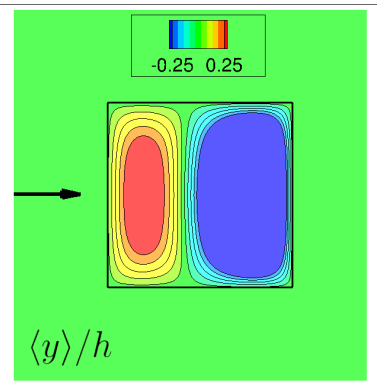

(b) time-mean panel deflection

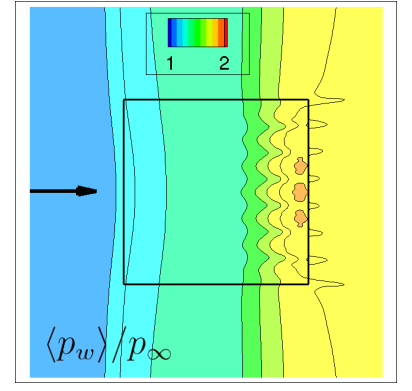

(c) time-mean wall pressure

Figure 11: POD modal energy content and the time-averages of the panel deflection and wall pressure (a) Energy contribution of POD modes (b) time-averaged panel deflection (b) time-averaged wall pressure.

1000 snapshots; however, due to optimality of the POD [7], only few modes comprise most of the energy. The figure (Fig. 11(a)) shows first 100 modes ordered based on their energy content for both the variables. Clearly, the first two modes of panel deflection and first ten modes of wall pressure contain $\approx 99 \%$ of the total energy. Thus, we retain only these modes, in addition to the time-averages of variables, for ROM predictions. Figure 11(b) and (c) show the time-averaged panel deflection and wall pressure profiles respectively, where the profiles are normalized by the panel thickness and free-stream pressure $\left(p_{\infty}\right)$ respectively. The timemean panel deflection profile exhibits a sinusoidal response (which corresponds to the mode 2,1 of panel deformation) due to the impinging shock, the wall pressure on the other hand (Fig. 11 c) indicates the typical increase across a shock.
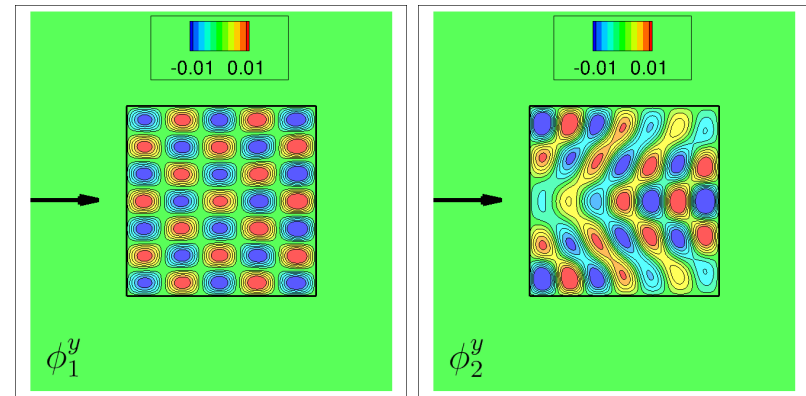

(a) POD space modes of panel deflection

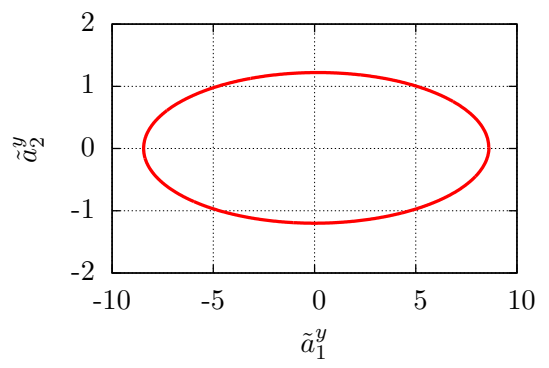

(b) POD time modes of panel deflection

Figure 12: First two POD modes of the panel deflection (a) POD space modes (b) POD time modes in terms of phase portrait. The arrows indicate flow direction.

The first two energy dominant POD modes for the panel deflection, Fig. 12 (a), indicate higher modes of panel vibration [49, where the first mode constitutes most energy; which is $\approx 97.43 \%$ as opposed to 
$\approx 1.90 \%$ for the second mode. The corresponding characteristic POD time modes that are extracted from the regular POD time modes are shown in Fig. 12(b) in terms of a phase portrait, where both the modes exhibit limit cycle oscillations $(\mathrm{LCO})$ with a time period of $\approx 0.7855$.

The energy dominant POD modes of the wall pressure are shown in Fig. 13. The first two POD space modes of wall pressure, Fig. 13(a), exhibit a correspondence with the first two POD modes of the panel deflection; where the mode shapes of wall pressure show undulations of approximately same wavenumbers in the streamwise and spanwise directions. However, the higher POD space modes of wall pressure exhibit undulations that correspond to the panel deflection modes in the spanwise direction only (Fig. 13 a). The SWBLI coupled with panel flutter results in flow transition, giving rise to the flow three-dimensionality and unsteadiness, mostly near the shock-foot and downstream regions (refer the 3-D flow in Fig. 10 b); consequently, the POD space modes of wall pressure extend approximately from the shock impingement location to the downstream end computational domain. The corresponding POD time modes are shown in Fig. 13(b) in terms of phase portraits, where LCO of the first seven modes are displayed. Furthermore, the phase portraits in Fig. 13(b) exhibit pairs of POD modes: $\left(\tilde{a}_{1}^{p_{w}}-\tilde{a}_{2}^{p_{w}}\right),\left(\tilde{a}_{3}^{p_{w}}-\tilde{a}_{4}^{p_{w}}\right)$ and $\left(\tilde{a}_{5}^{p_{w}}-\tilde{a}_{6}^{p_{w}}\right)$ etc., where the modes from a pair show a phase shift with respect to the first POD mode $\tilde{a}_{1}^{p_{w}}$ for the same value of time period. The presence of POD mode pairs indicates organized flow structures of the transitional SWBLI, as the boundary layer is not developed to a fully turbulent state.

\subsubsection{ROM solution}

As noted before, only first two modes of the panel deflection and first ten modes of wall pressure constitute $\approx 99 \%$ of the total energy, forming a reduced order POD basis. The POD reduced basis comprise the reduced number of POD modes for all reference cases listed in Table 3, and an off-reference case solution can be readily obtained by the ROM procedure shown in Fig. 7. We consider an off-reference case with the panel thickness of $h^{*}=0.010$. In order to perform the linear interpolation defined in Sec. 2.2, two reference cases that encompasses the off-reference case are selected, which include: $h^{*}=0.008$ and $h^{*}=0.016$. The interpolation leads to a reduced POD basis for $h^{*}=0.010$, which includes the POD space and time modes as well as the time-averaged variables. Figure 14 shows the interpolated POD modes in comparison with the high-fidelity POD modes at the same value of panel thickness $\left(h^{*}=0.010\right)$. The first two energy dominant POD modes for the panel deflection, $\phi_{1}^{y}$ and $\phi_{2}^{y}$, are shown are in Fig. 14(a) and Fig. 14(b), where the interpolated modes are almost identical to the high-fidelity modes. The interpolated modes follow the sign convention of any one reference case, here they follow $h^{*}=0.008$. Although a large difference in the reference cases can introduce non-linearities and the linear interpolation may result in higher numerical error, the dominant POD modes appear to be consistent over the considered range of panel thickness. Xie et al. 52] made similar observation while developing a ROM for a nonlinear aeroelastic flutter based on their prior study [53], where a set of POD modes was used for various cases with different flutter parameter 

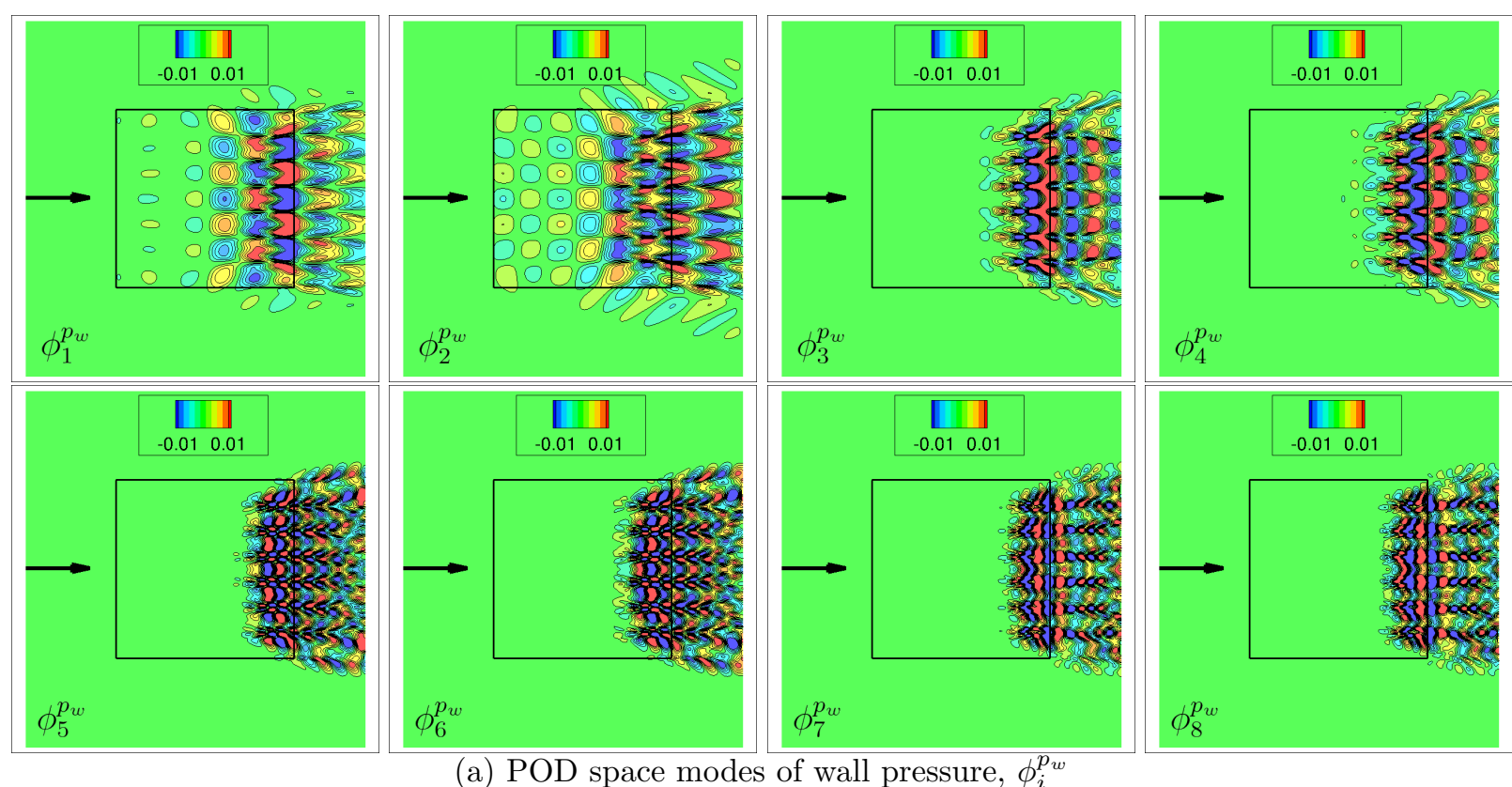

(a) POD space modes of wall pressure, $\phi_{i}^{p_{w}}$
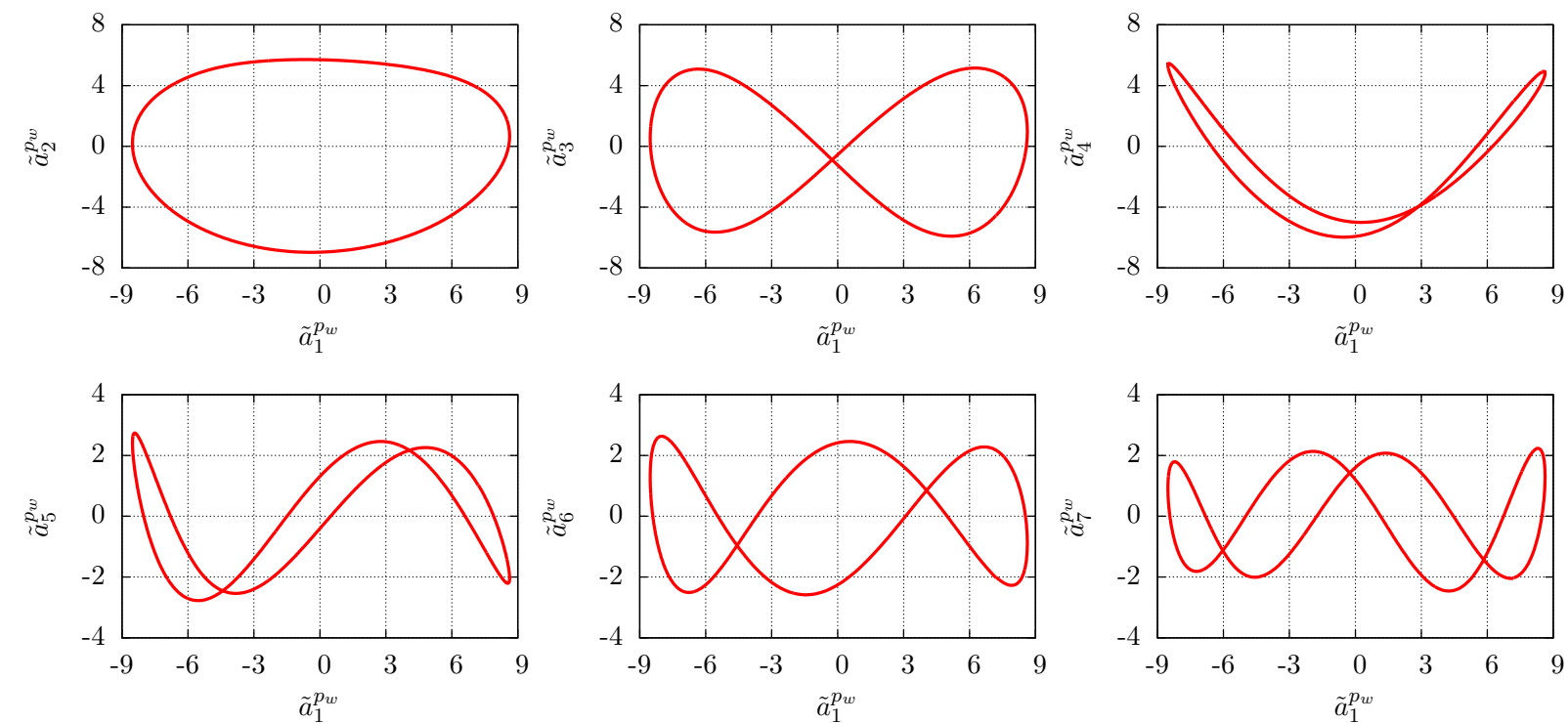

(b) POD temporal modes for the wall pressure, $\tilde{a}_{i}^{p_{w}}$

Figure 13: Energy dominant POD space and characteristic time modes of wall pressure. (a) POD space modes $\phi_{i}^{p_{w}}$ (b) POD time modes $\tilde{a}_{i}^{p_{w}}$. The arrows indicate flow direction. 
and aspect ratios. The POD modes for the wall pressure are compared between the high-fidelity simulation and ROM in Fig. 14(b), where the first $\left(\phi_{1}^{p_{w}}\right)$ and eighth $\left(\phi_{8}^{p_{w}}\right)$ POD space modes are compared, showing a good match even for the low energy eighth mode $\left(\phi_{8}^{p_{w}}\right)$.
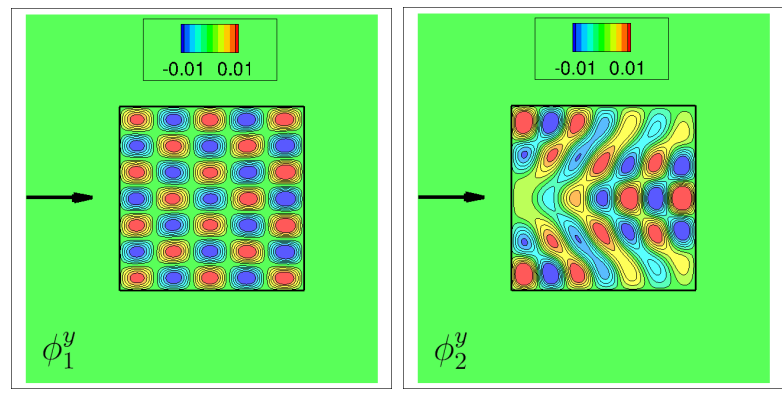

(a) HF modes

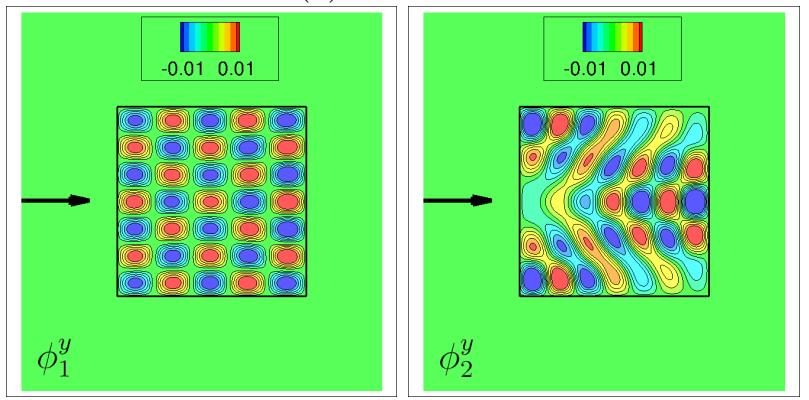

(b) interpolated modes

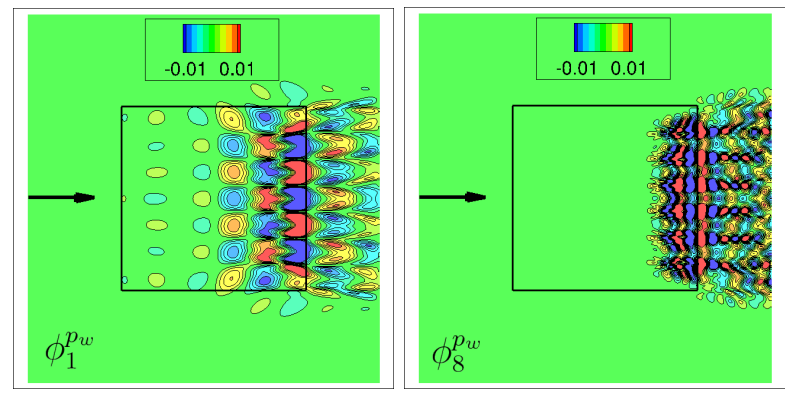

(c) HF modes

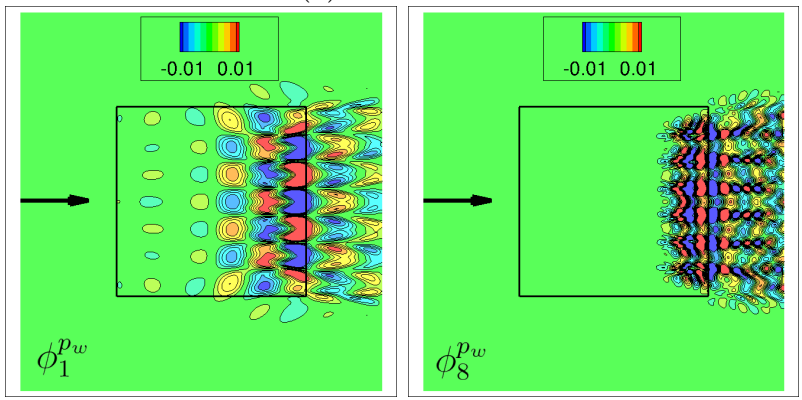

(d) interpolated modes

Figure 14: Comparison between the high-fidelity and interpolated POD space modes from reduced POD basis for $h^{*}=0.010$ (a) high-fidelity modes for panel deflection (b) interpolated modes for panel deflection (c) high-fidelity modes for wall pressure (d) interpolated modes for wall pressure. The arrows indicate flow direction.

The space-invariant POD reduced basis, which consists of the characteristics POD time modes, is also interpolated by using the procedure in Sec. 2.2. The linear interpolation of the first two and first ten POD modes for the panel deflection and wall pressure is performed for the off-reference case of $h^{*}=0.010$ by using the two reference cases: $h^{*}=0.008$ and $h^{*}=0.016$, similar to the POD space modes and time averages. Figure 15 shows linearly interpolated characteristic POD time modes for the panel deflection and wall pressure for $h^{*}=0.010$ in comparison with the high-fidelity solutions. As opposed to the POD space modes, the temporal modes exhibit non-linearity in their amplitudes and phase values for the change in controlling parameter, leading to higher differences between the high-fidelity solution and ROM interpolation. The phase portrait of the first two POD modes of the panel deflection is shown in Fig. 15(a), comparing the high-fidelity and ROM LCO; where the interpolated modes, to some degree, under predict the amplitudes. The interpolation of the second, third and eighth modes against the first mode of the wall pressure is presented in Fig. 15(a), (b) and (c) respectively, indicating a good match even for the higher mode $\tilde{a}_{8}^{p_{w}}$.

The ROM results are compared with high-fidelity solution in terms of the POD modal energy and panel 


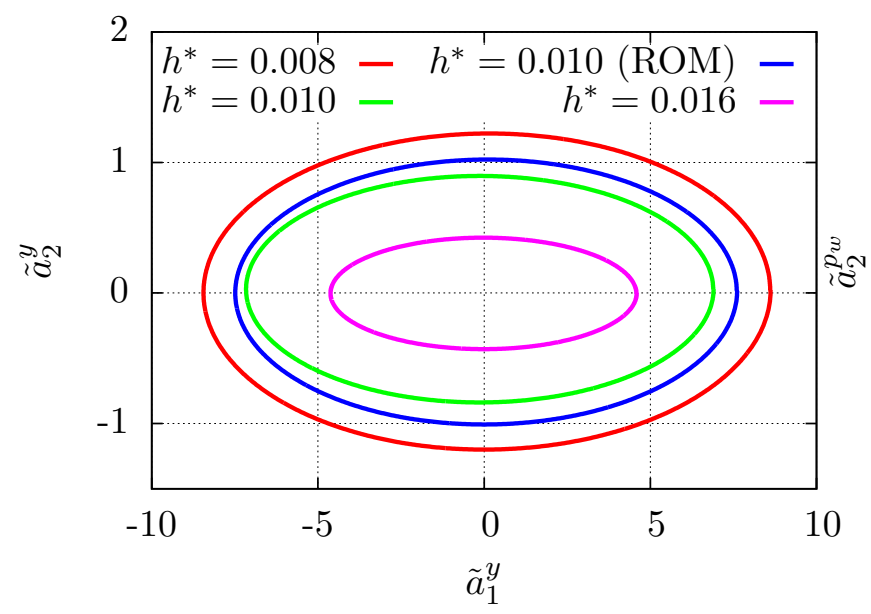

(a)

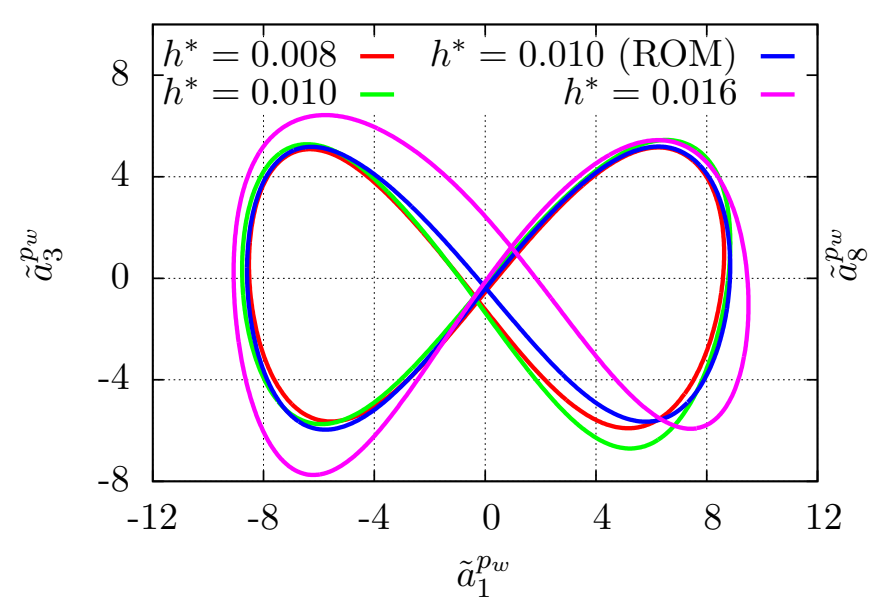

(c)

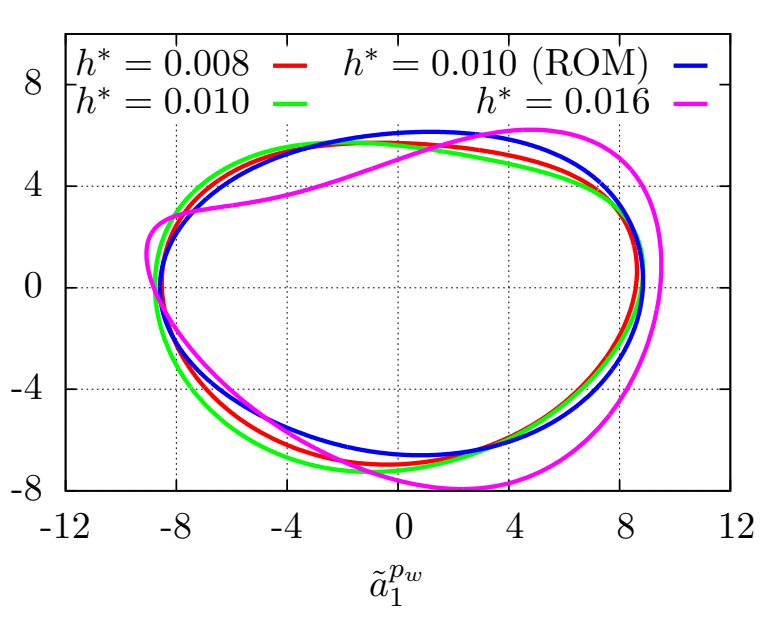

(b)

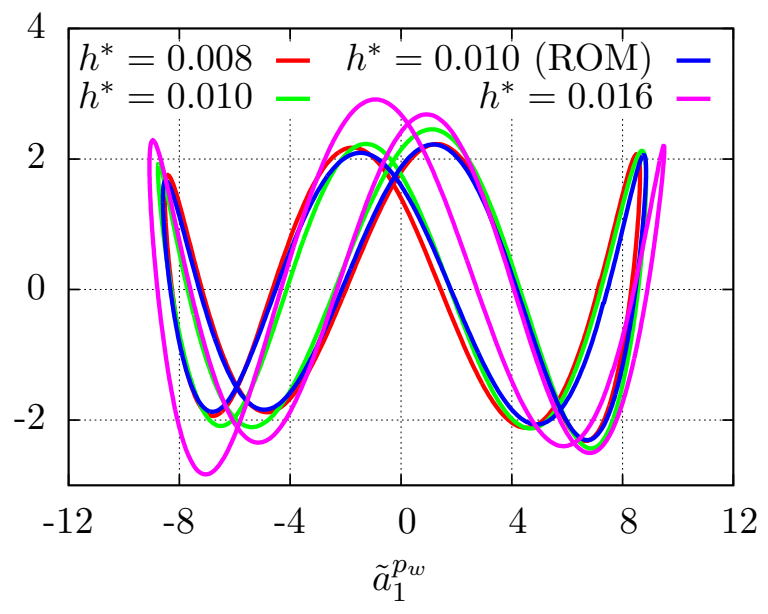

(d)

Figure 15: Interpolation of characteristic POD time modes $h^{*}=0.010$. (a) phase portrait of first and second modes of panel deflection (b) phase portrait of first and second modes of wall pressure (c) phase portrait of first and third modes of wall pressure (d) phase portrait of first and eighth modes of wall pressure 


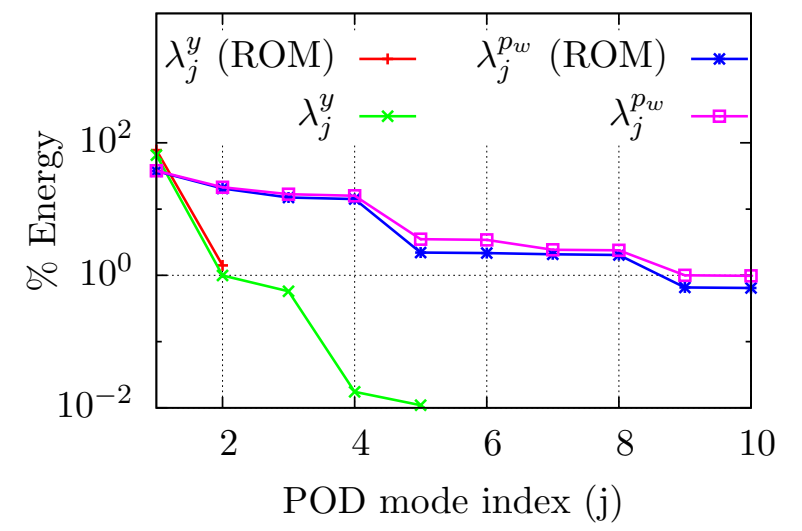

(a) POD modal energy

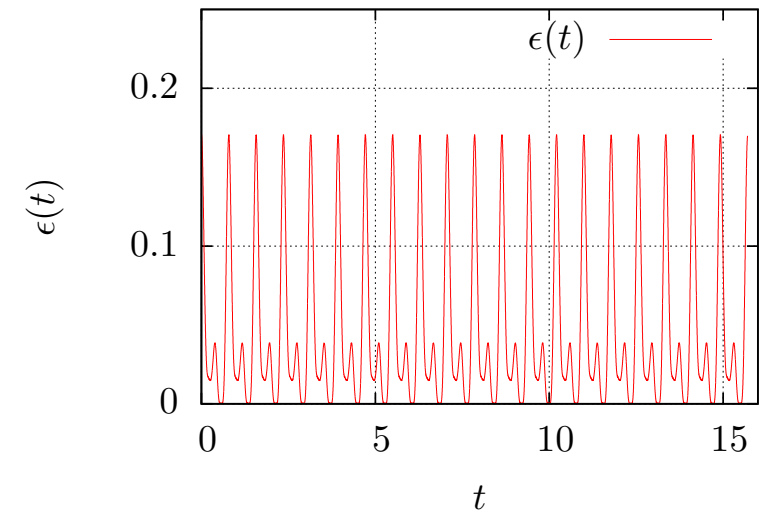

(a) instantaneous error

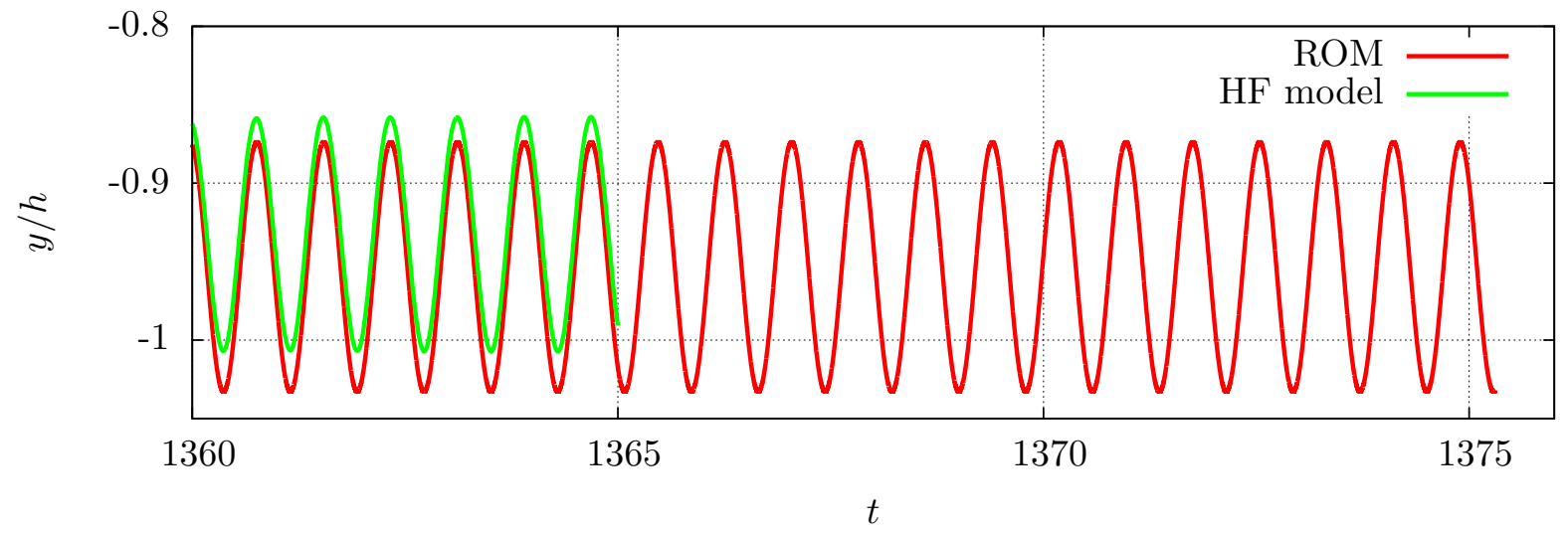

(c) panel flutter response at a probe location

Figure 16: ROM predictions and error $h^{*}=0.010$. (a) POD modal energy of reduced POD basis (b) time evolution of total error (refer Eq. 18) (c) comparison of flutter response between the HF and ROM in term of panel deflection at the 3/4 chord length location 
flutter response at a probe location in Fig. 16. The POD modal energy contribution, Fig. 16 (a), for the ROM is in good agreement with the high-fidelity modes for both the panel deflection and wall pressure, where the reduced basis comprise $\approx 99 \%$ of the total energy. The error in ROM prediction based on Eq. 18 is shown in Fig. 16(a), where the time evolving error exhibits LCO with a maximum value of $\approx 0.17$ in a cycle. As discussed in Shinde et al. 23], the Galerkin-free POD ROM approach is unconditionally stable, where the error $\epsilon(t)$ remains in bounds for values of $t$. The panel flutter response in terms of the panel deflection at the $3 / 4$ chord length is compared in Fig. 16(c), where the ROM predictions closely follow the high-fidelity solution. The time-averaged panel deflection and its standard deviation are $y_{m} / h=0.9535$ and $y_{S D} / h=0.0564$ against the high-fidelity simulation values of $y_{m} / h=0.9331$ and $y_{S D} / h=0.0523$ respectively.

A high-fidelity simulation costs $\approx 7500$ hours of processor time in order to obtain a statistically stationary solution. The presented Galerkin-free procedure predicts the reduced order solution in $\approx 10$ seconds, substantially reducing the computational cost; which is also due to the partial selection of the computational domain and solution state variables while constructing the ROM.

\section{Conclusion}

A Galerkin-free model reduction of fluid-structure interactions based on proper orthogonal decomposition is presented. The computational mesh deformation is considered as a part of the solution state vector while constructing the reduced POD basis. The approach is demonstrated by using two case studies: a vortexinduced vibration of a cylinder at low Reynolds number and a shock boundary layer induced panel flutter, which comprise the two-dimensional laminar and three-dimensional transitional flows respectively. The energy dominant POD time modes must exhibit a periodic response in order to extract the characteristic POD time modes. The periodicity assumption holds for both the two dimensional VIV of cylinder and threedimensional panel flutter cases. The presented ROM procedure is clean and robust in terms of handling large mesh deformations, in addition to a significant reduction of computing cost, which is enticing for the flow/flutter control applications.

\section{Acknowledgement}

The authors acknowledge Centre National de la Recherche Scientifique (CNRS) for facilitating the work via Agence Nationale de la Recherche (ANR) project Baresafe. The authors acknowledge Prof. Datta Gaitonde for providing access to the high-fidelity database of shock boundary layer induced panel flutter. 


\section{References}

1. Lucia DJ, Beran PS, Silva WA. Reduced-order modeling: new approaches for computational physics. Progress in Aerospace Sciences 2004;40(1):51-117.

2. Moore B. Principal component analysis in linear systems: Controllability, observability, and model reduction. Automatic Control, IEEE Transactions on 1981;26(1):17-32.

3. Li D, Tan S, McGaughy B. Etbr: Extended truncated balanced realization method for on-chip power grid network analysis. In: Design, Automation and Test in Europe, 2008. 2008:432-7.

4. Glover K. All optimal hankel-norm approximations of linear multivariable systems and their $l^{\infty}$-error bounds. International Journal of Control 1984;39(6):1115-93.

5. Lumley JL. The Structure of Inhomogeneous Turbulent Flows. In: Yaglom AM, Tatarski VI, eds. Atmospheric turbulence and radio propagation. Moscow: Nauka; 1967:166-78.

6. Sirovich L. Turbulence and the dynamics of coherent structures. part i: Coherent structures. Quaterly of Applied Mathematics 1987;XLV(3):561-71.

7. Holmes P, Berkooz G, Lumley JL. Turbulence, dynamical systems and the unreasonable effectiveness of empirical eigenfunctions. In: Proceedings of the International Congress of Mathematicians, Kyoto. 1990:1607-17.

8. Dowell EH, Hall KC. Modeling of fluid-structure interaction. Annual Review of Fluid Mechanics 2001;33(1):445-90.

9. Beran PS, Lucia DJ, Pettit CL. Reduced-order modelling of limit-cycle oscillation for aeroelastic systems. Journal of Fluids and Structures 2004;19(5):575-90.

10. Vierendeels J, Lanoye L, Degroote J, Verdonck P. Implicit coupling of partitioned fluid-structure interaction problems with reduced order models. Comput Struct 2007;85(11-14):970-6.

11. Kalashnikova I, Barone M, Brake M. A stable galerkin reduced order model for coupled fluid-structure interaction problems. International Journal for Numerical Methods in Engineering 2013;95(2):121-44.

12. Amsallem D, Tezaur R, Farhat C. Real-time solution of linear computational problems using databases of parametric reduced-order models with arbitrary underlying meshes. Journal of Computational Physics 2016;326:373-97.

13. Silva WA, Bartels RE. Development of reduced-order models for aeroelastic analysis and flutter prediction using the cfl3dv6. 0 code. Journal of Fluids and Structures 2004;19(6):729-45.

14. Raveh DE. Computational-fluid-dynamics-based aeroelastic analysis and structural design opimization - a researcher's perspective. Computer Methods in Applied Mechanics and Engineering 2005;194(30-33):3453-71. Structural and Design Optimization.

15. Colciago C, Deparis S, Quarteroni A. Comparisons between reduced order models and full 3d models for fluid-structure interaction problems in haemodynamics. Journal of Computational and Applied Mathematics 2014;265(0):120-38. Current Trends and Progresses in Scientific Computation Dedicated to Professor Ben-yu Guo on His 70th Birthday.

16. Gerbeau JF, Vidrascu M. A quasi-newton algorithm based on a reduced model for fluid-structure interaction problems in blood flows. ESAIM: Mathematical Modelling and Numerical Analysis-Modélisation Mathématique et Analyse Numérique $2003 ; 37(4): 631-47$.

17. Anttonen J, King P, Beran P. Pod-based reduced-order models with deforming grids. Mathematical and Computer Modelling 2003;38(1):41-62.

18. Anttonen JS, King PI, Beran PS. Applications of multi-pod to a pitching and plunging airfoil. Mathematical and Computer Modelling 2005;42(3):245-59.

19. Bourguet R, Braza M, Dervieux A. Reduced-order modeling of transonic flows around an airfoil submitted to small deformations. Journal of Computational Physics 2011;230(1):159 -84.

20. Iemma U, Gennaretti M. Reduced-order modeling for linearized aeroelasticity of fixed wings in transonic flight. Journal of fluids and structures 2005;21(3):243-55. 
21. Bui-Thanh T, Willcox K, Ghattas O, van Bloemen Waanders B. Goal-oriented, model-constrained optimization for reduction of large-scale systems. Journal of Computational Physics 2007;224(2):880-96.

22. Lassila T, Rozza G. Reduced formulation of a steady fluid-structure interaction problem with parametric coupling. arXiv preprint arXiv:10053384 2010;

23. Shinde V, Longatte E, Baj F, Hoarau Y, Braza M. A galerkin-free model reduction approach for the navier-stokes equations. Journal of Computational Physics 2016;309:148-63.

24. Morzynski M, Stankiewicz W, Noack BR, Thiele F, King R, Tadmor G. Generalized mean-field model for flow control using a continuous mode interpolation. In: Proceedings of the Third AIAA Flow Control Conference. 2006:1-11.

25. Balajewicz M, Dowell E. Stabilization of projection-based reduced order models of the navier-stokes. Nonlinear Dynamics $2012 ; 70(2): 1619-32$.

26. Demmel J. Applied Numerical Linear Algebra. Society for Industrial and Applied Mathematics; 1997.

27. Shinde VJ, McNamara JJ, Gaitonde DV. Effect of structural parameters on shock wave boundary layer induced panel flutter. In: AIAA Aviation 2019 Forum. 2019:3716.

28. Hoarau Y, Pena D, Vos JB, Charbonier D, Gehri A, Braza M, Deloze T, Laurendeau E. Recent developments of the navier stokes multi block (nsmb) cfd solver. In: 54th AIAA Aerospace Sciences Meeting. 2016:2056.

29. Bourguet R, Braza M, Harran G, El Akoury R. Anisotropic organised eddy simulation for the prediction of non-equilibrium turbulent flows around bodies. Journal of Fluids and Structures 2008;24(8):1240-51.

30. Vos J, Rizzi A, Corjon A, Chaput E, Soinne E. Recent advances in aerodynamics inside the nsmb (navier-stokes multi block) consortium. In: 36th AIAA Aerospace Sciences Meeting and Exhibit, Reno, NV, USA. AIAA; 1998:98-0225.

31. Turkel E, Vatsa VN, Radespiel R. Preconditioning methods for low-speed flows. National Aeronautics and Space Administration 1996;NASA CR 201605.

32. Erlicher S, Bonaventura L, Bursi OS. The analysis of the generalized- $\alpha$ method for non-linear dynamic problems. Computational Mechanics 2002;28(2):83-104.

33. Williamson C, Govardhan R. Vortex-induced vibrations. Annu Rev Fluid Mech 2004;36:413-55.

34. Shinde V, Longatte E, Baj F, Braza M. A theoretical model of fluidelastic instability in tube arrays. Nuclear Engineering and Design 2018;337:406-18.

35. Khalak A, Williamson C. Fluid forces and dynamics of a hydroelastic structure with very low mass and damping. Journal of Fluids and Structures 1997;11(8):973-82.

36. Khalak A, Williamson C. Motions, forces and mode transitions in vortex-induced vibrations at low mass-damping. Journal of fluids and Structures 1999;13(7):813-51.

37. Tadmor G, Lehmann O, Noack BR, Morzyński M. Galerkin models enhancements for flow control. In: Reduced-Order Modelling for Flow Control. Springer; 2011:151-252.

38. Bui-Thanh T, Damodaran M, Willcox K. Proper orthogonal decomposition extensions for parametric applications in compressible aerodynamics. In: Proceedings of 21st AIAA Applied Aerodynamics Conference, Orlando, Florida. 2003:111.

39. Lieu T, Farhat C, Lesoinne M. Reduced-order fluid/structure modeling of a complete aircraft configuration. Computer methods in applied mechanics and engineering 2006;195(41):5730-42.

40. Amsallem D, Farhat C. Interpolation method for adapting reduced-order models and application to aeroelasticity. $A I A A$ journal 2008;46(7):1803-13.

41. Amsallem D, Farhat C. An online method for interpolating linear parametric reduced-order models. SIAM Journal on Scientific Computing 2011;33(5):2169-98.

42. Liberge E, Hamdouni A. Reduced order modelling method via proper orthogonal decomposition (pod) for flow around an oscillating cylinder. Journal of Fluids and Structures 2010;26(2):292-311. 
43. Shinde V. Fluidelastic instability in heat exchanger tube arrays and a galerkin-free model reduction of multiphysics systems. Ph.D. thesis; Ecole Polytechnique; 2015.

44. Rempfer D, Fasel HF. Dynamics of three-dimensional coherent structures in a flat-plate boundary layer. Journal of Fluid Mechanics 1994;275:257-83.

45. Gaitonde DV, Visbal MR. Padé-type higher-order boundary filters for the navier-stokes equations. AIAA journal 2000;38(11):2103-12.

46. Visbal MR, Gaitonde DV. High-order-accurate methods for complex unsteady subsonic flows. AIAA journal 1999;37(10):1231-9.

47. Visbal MR, Gaitonde DV. On the use of higher-order finite-difference schemes on curvilinear and deforming meshes. Journal of Computational Physics 2002;181(1):155-85.

48. Visbal M, Gordnier R. Numerical simulation of the interaction of a transitional boundary layer with a 2-d flexible panel in the subsonic regime. Journal of fluids and structures 2004;19(7):881-903.

49. Shinde V, McNamara JJ, Gaitonde DV, Barnes CJ, Visbal MR. Panel flutter induced by transitional shock wave boundary layer interaction. In: 2018 Fluid Dynamics Conference. 2018:3548.

50. Shinde V, Gaitonde DV, McNamara JJ. Control of transitional shock boundary layer interaction using surface morphing. In: AIAA Scitech 2019 Forum. 2019:1895.

51. Gordnier RE, Visbal MR. Development of a three-dimensional viscous aeroelastic solver for nonlinear panel flutter. Journal of fluids and structures 2002;16(4):497-527.

52. Xie D, Xu M, Dowell EH. Projection-free proper orthogonal decomposition method for a cantilever plate in supersonic flow. Journal of Sound and Vibration 2014;333(23):6190-208.

53. Xie D, Xu M, Dowell EH. Proper orthogonal decomposition reduced-order model for nonlinear aeroelastic oscillations. AIAA journal 2014;52(2):229-41. 